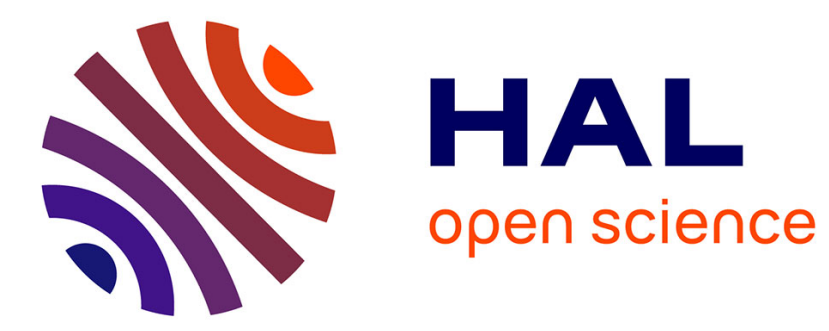

\title{
Generation of coherent wavefronts and images inside laser resonators
}

Michel Vampouille, Bernard Colombeau, Vincent Kermène, P. Martin, Claude Froehly

\section{- To cite this version:}

Michel Vampouille, Bernard Colombeau, Vincent Kermène, P. Martin, Claude Froehly. Generation of coherent wavefronts and images inside laser resonators. Journal de Physique III, 1993, 3 (7), pp.1369-1380. 10.1051/jp3:1993205 . jpa-00249005

\section{HAL Id: jpa-00249005 https://hal.science/jpa-00249005}

Submitted on 1 Jan 1993

HAL is a multi-disciplinary open access archive for the deposit and dissemination of scientific research documents, whether they are published or not. The documents may come from teaching and research institutions in France or abroad, or from public or private research centers.
L'archive ouverte pluridisciplinaire HAL, est destinée au dépôt et à la diffusion de documents scientifiques de niveau recherche, publiés ou non, émanant des établissements d'enseignement et de recherche français ou étrangers, des laboratoires publics ou privés. 


\title{
Generation of coherent wavefronts and images inside laser resonators
}

\author{
M. Vampouille, B. Colombeau, V. Kermene, P. Martin and C. Froehly \\ IRCOM, URA CNRS 356, 123 rue Albert Thomas, 87060 Limoges, France
}

(Received 3 December 1992, accepted 21 Aprll 1993)

\begin{abstract}
Résumé. - Nous décrivons un nouveau type de filtrage des modes d'un laser basé sur l'utilisation de deux filtres spatiaux complémentaires situés contre les miroirs plans d'un résonateur confocal à lentille. Nous rapportons la synthèse de deux ondes particulières dans un laser à YAG/Nd : une surface d'onde modulée sinusoïdalement et un faisceau uniphase, de profil spatial plat et de divergence minimale. L'évolution temporelle du faisceau pendant la phase de construction est aussi rapportée. Ce procédé est un nouveau type de reconstruction de front d'onde au même titre que l'holographie, mais sans référence.
\end{abstract}

\begin{abstract}
We describe a new type of laser mode filtering using two complementary spatial filters located against the plane mirrors of a confocal lens resonator. With Nd/YAG as amplifying medium. we report the synthesis of two particular waves : a sinusoidally modulated wavefront, and a uniphase beam with a flat spatial profile and minimal divergence. The temporal evolution of the beam under construction is also recorded. The process is a new type of wavefront reconstruction, like holography, but without reference.
\end{abstract}

\section{Introduction.}

The «phase problem » can be found in many fields : diffraction optics, electron microscopy and X-ray diffraction, stellar interferometry.. Considerable effort has been devoted to the development of algorithms yielding numerical solutions. Although there have been some successes, the problem remains difficult and the difficulty depends on the available information.

In the following, we consider the classical problem of recovering the amplitude and phase of a light wave when we only know its modulus in two Fourier planes; and if the solution does exist, is this the only possible one?

From an analytical point of view, this problem has been explained at length by Barakat and Newsam [1, 2] in a mathematical review. In a simplified paper [3], these authors in particular 
say that the solution is unique if the function $f$ and its Fourier transform FT $(f)$ are bidimensional and analytic, and if FT $(f)$ vanishes outside a bounded interval. From a numerical point of view, a lot of algorithms have been found by several authors. It is important to note that most of these algorithms are not based on rigorous mathematical principles and generate solutions fluctuating around an average value. From an experimental point of view, as far as we know, there have been up to now few experiments which really reconstructed a complex function from the knowledge of its modulus and the modulus of its Fourier transform [4].

The aim of this article is to show experimentally the build-up of wavefronts inside a laser using data concerning the intensity into Fourier planes. These data are placed in the resonator by means of two complementary binary masks. We have decided to describe the series of iterative operations undergone by the light in the resonator by referring to the Gerchberg and Saxton's iterative algorithm often used for the phase retrieval. We then report experimental results concerning two particular waves : a sinusoidally modulated wavefront, and a uniphase beam with a flat spatial profile and minimal divergence. Finally, we study the temporal evolution of the beam under construction.

This paper is concerned with the works which study the control of the transversal spatial structure of laser modes, obtained by placing different obstacles in the cavity : one or several diaphragms, tightened wires, binary masks, Gaussian mirrors, or non linear components.. [5$10]$.

\section{Gerchberg-Saxton algorithm.}

To understand the principle of the experiment that we have implemented, let us first briefly explain the algorithm derived from Gerchberg and Saxton [11]. This numerical algorithm finds the phase $\varphi(x)$ of a complex function $f(x)$, of which only the moduli $\|f(x)\|$ and $\|\mathrm{FT}[f(x)]\|$ are known. It works by successive perturbations on all the samples of $f(x)$ and FT $[f(x)]$ (see Fig. 1).

We start with any phase function $\mathrm{e}^{/ \phi(1)}$ and we build $g(x)=\|f(x)\| \mathrm{e}^{/ \phi(1)}$ with the known modulus $\|f(x)\|$. We take its Fourier transform (1) and we replace (2) the modulus of this new function by $\|F(u)\|$ which is also known. We obtain $G^{\prime}(u)=\|F(u)\| \mathrm{e}^{\prime \varphi(u)}$ and we take the inverse Fourier transform of this function (3). The modulus of this new function will then be replaced (4) by the known modulus $\|f(x)\|$. At each step, the phase term will be retained, whereas the computed modulus will be replaced by its known value. The four operations will be iterated until they yield converging solutions.

Our new holographic principle will consist in just the analogical transposition of this algorithmic process.

\section{Analogical transposition of the algorithm.}

3.1 FIRST TRANSPOSITION IN LINE (see Fig. 2). - The analogical Fourier transform is achieved by propagation of a monochromatic wave between front and back-focal planes of a converging lens. The sequence of Fourier transforms is made with a sequence of several lenses $\mathrm{L}_{l}$ having their focal planes at the same place. A set of $4 n$ lenses is necessary to synthesize a non-symmetrical complex wave. Conservation of phase terms and the processing of moduli exchange after each F.T. are achieved by two bidimensional masks, the real transparency of which is deduced from $\|f(x, y)\|$ and $\|F(u, v)\|$ respectively. In the algorithm, the functions are normalized at each iteration: in the analogical process, the losses caused by the masks must be compensated by the gain of one or several amplifying materials. 


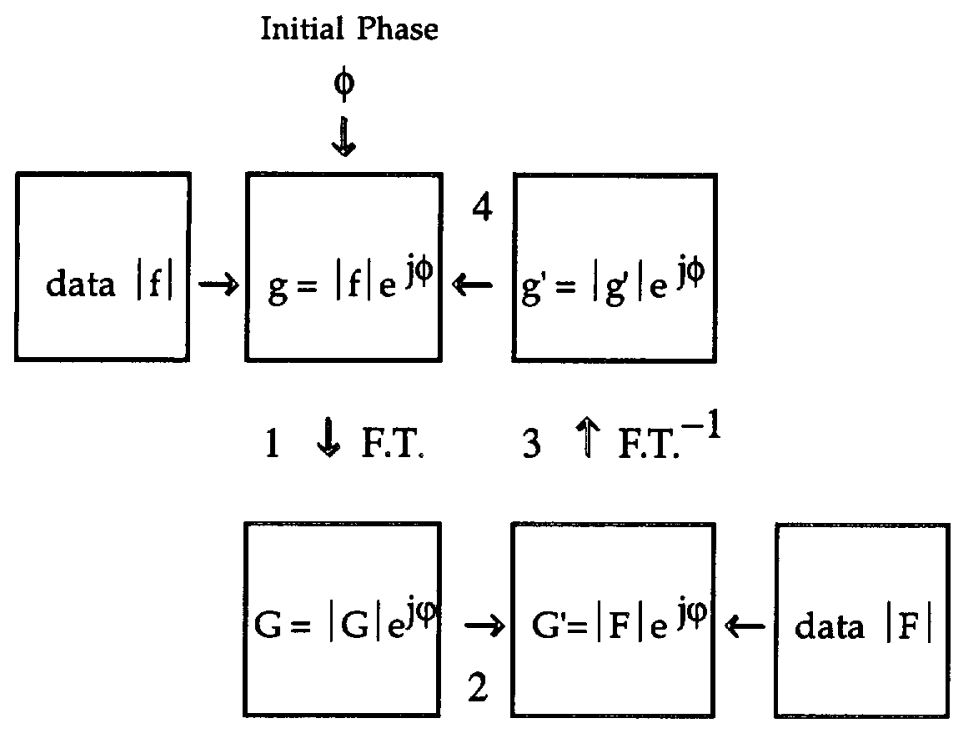

Fig. 1. - Gerchberg-Saxton algorithm.

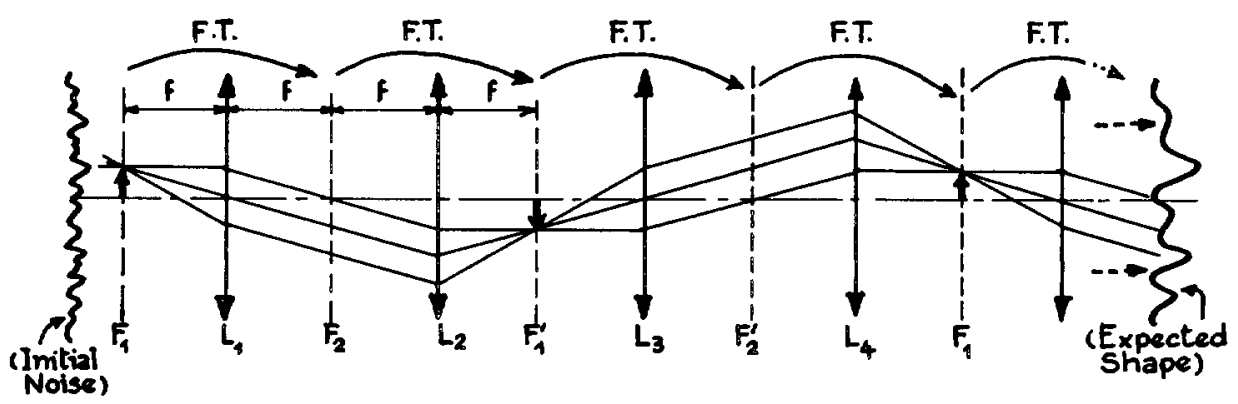

1. F.T. $\neq \mathrm{F} \cdot \mathrm{T}^{-1} . \Rightarrow 4 \mathrm{n}$ lenses required for synthesis of unsymmetrical shapes.

2. Substitution of field amplitudes ( moduli substitution $») \Rightarrow$ approximated by filtering through real transparencies.

3. Filtering losses $\Rightarrow$ compensation by LASER amplification.

Fig. 2. - Analogical transposition of the algorithm.

We must notice an important difference between the algorithm and our analogical transposition : the moduli are not replaced by the exact solutions, but multiplied by the real transparency of the masks. In practice, these masks are of binary transmission. Thus, the constraints working on the wave are less restrictive than in the Gerchberg-Saxton algorithm ; sometimes, they are not sufficient to obtain the convergence.

3.2 SECOND TRANSPOSITION : USE OF RING RESONATORS. - It is clear that the above sequence of operations may be performed inside a ring only containing four converging lenses and four plane mirrors, see figure 3. An amplifying material (A.M.) transforms this ring into a special ring laser cavity equipped with two pairs of binary transparency masks $F_{1}$ and $F_{2}$. This cavity will be able to synthesize complex waves of any shape presenting no particular symmetry. We are presently working on such resonators. 


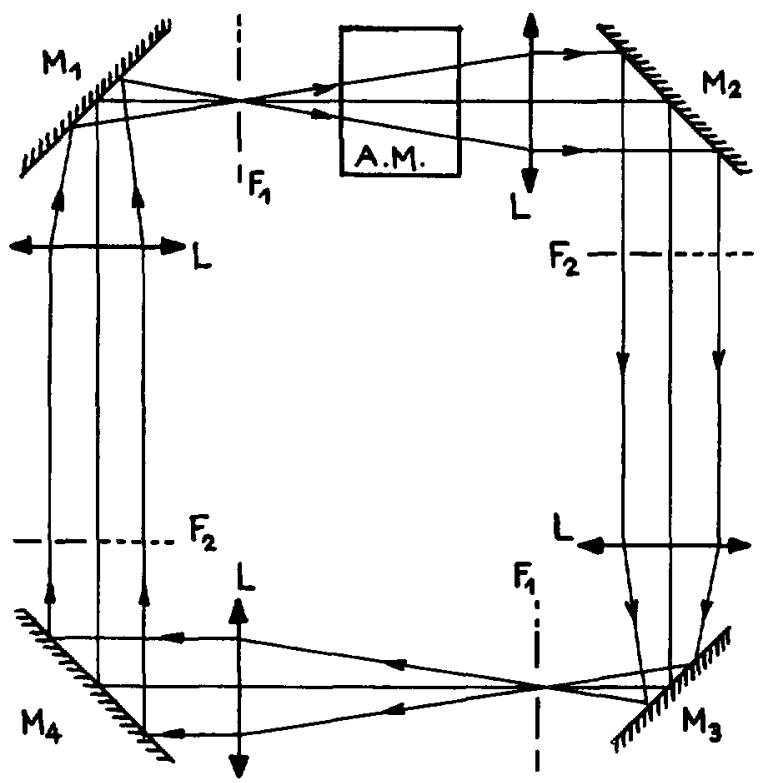

Fig. 3. - Ring laser resonator with 4 converging lenses and 2 pairs of masks to synthesize any complex waves.

3.3 THIRD TRANSPOSITION : FABRY-PÉROT CAVITY ONLY VALID FOR SYMMETRICAL REAL WAVES. - Firstly, we wanted to test the validity of our process with the synthesis of symmetrical real waves. For this purpose, we built a simpler resonator, of the confocal FabryPérot type, figure 4. The Fabry-Pérot confocal resonator contains a single converging lens $L$ and two plane mirrors $M_{1}, M_{2}$, located in its focal planes. The binary masks $F_{1}$ and $\mathrm{M}_{2}$ are located very close to the mirrors or even engraved on them. The amplifier is a YAG/Nd rod of $7 \mathrm{~mm}$ diameter, flash pumped at the repetition rate of $5 \mathrm{~Hz}$.

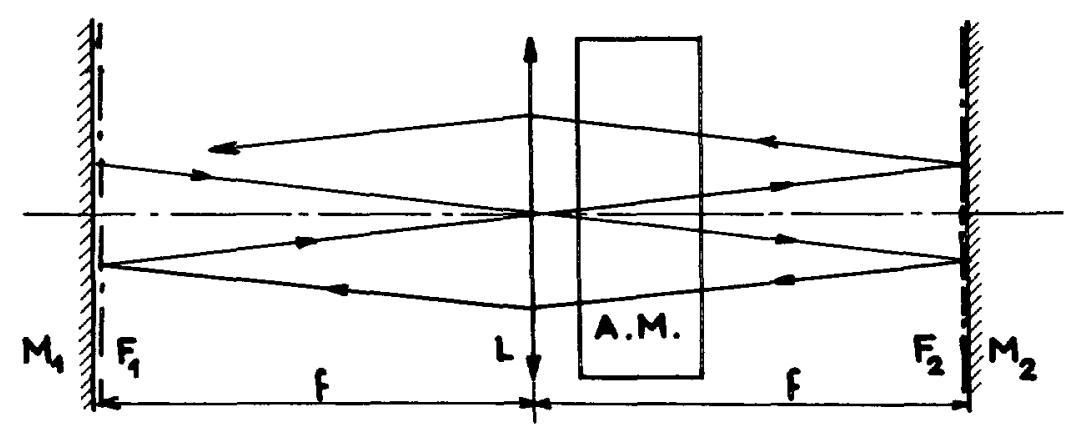

Fig. 4. - Confocal laser resonator, with a pair of spatial filters $F_{1}$ and $F_{2}$. The location of intensity zeroes, determined by setting binary masks $F_{1}, F_{2}$ in each of the Fourier conjugate focal planes of $L$ may, in some situations, be a strong enough constraint for inducing convergence of field distributions towards an unique solution. 


\section{Experimental results.}

The first light field that we synthesized was a spatially sinusoidal amplitude distribution with $\pi$ phase shifts at each half-period $p$ of the sine wave.

One of the masks consists in two small circular holes drilled through an opaque screen, separated from each other by a length $\lambda f / p$, reciprocal to the sinus periodicity $p$ ( $\lambda=$ wavelength, $f=$ focal length of the lens). The other mask is just a sequence of opaque wires (grid) accurately located at the positions of the intensity minimums of the sinus function to be generated (see Fig. 5). The pictures (Fig. 6) presented here were recorded by a spatial beam analyser producing a pictorial representation of the different energy levels in a three dimensional frame. The cavity works in the free-running regime, just above the laser emission threshold. In the grid plane (Fig. 6a), we can see the expected sinusoidal fringe pattern. In the far field, (Fig. 6b), we can observe two bright spots propagating with the angular spacing of the spatial frequences $\pm p / \lambda f$. The presence of these two spots demonstrates that the amplitude in the near field is really the expected sine wave function, rather than, for instance a (sinus) ${ }^{2}$ amplitude distribution. In this latter case, the different phases in the near field would keep the same sign. thus generating three spots instead of the two observed.

Moreover, if we remove the grid, the time integrated illumination in the near field looks like an almost uniform background : time resolved observation of the pulse demonstrates that the sinusoidal fringes exist at any time, but as they move randomly during the free running laser emission, they give rise to a statistically constant illumination. The masks achieve the phase locking of spatial modes which can coexist inside the laser cavity.

After these first results, we wanted to synthesize a square limited plane wave (constant illumination and phase across a square plane aperture) [12-14]. Such a laser wave is very interesting for many applications : drilling, soldering, surface treatment, information storage.. Here, we find the same cavity (see Fig. 7). Only the masks are different. The first one is a square aperture, of width a, cut in a black screen, the other one is a grid made of opaque wires coinciding with the zeroes of the bidimensional $\sin (\pi a r / \lambda f) /(\pi a x / \lambda f) . \sin (\pi a y / \lambda f) /$ $(\pi a y / \lambda f)$ function. These wires are engraved on the output mirror by a chemical process.

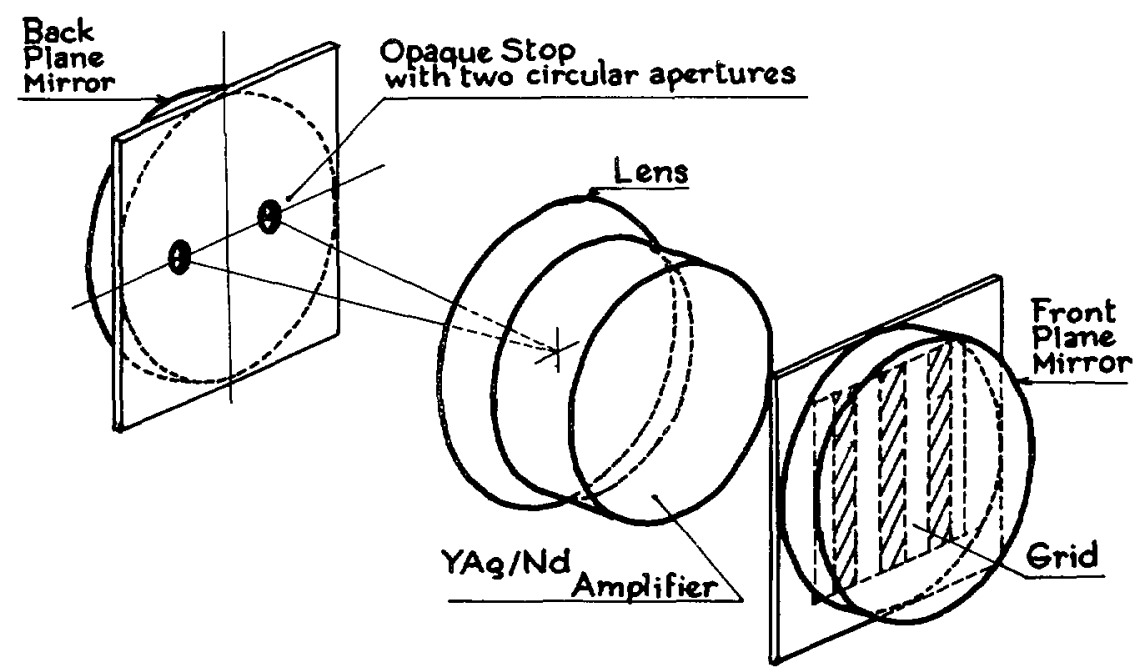

Fig. 5. - First experimental synthesis of a sinusoidal wave. 


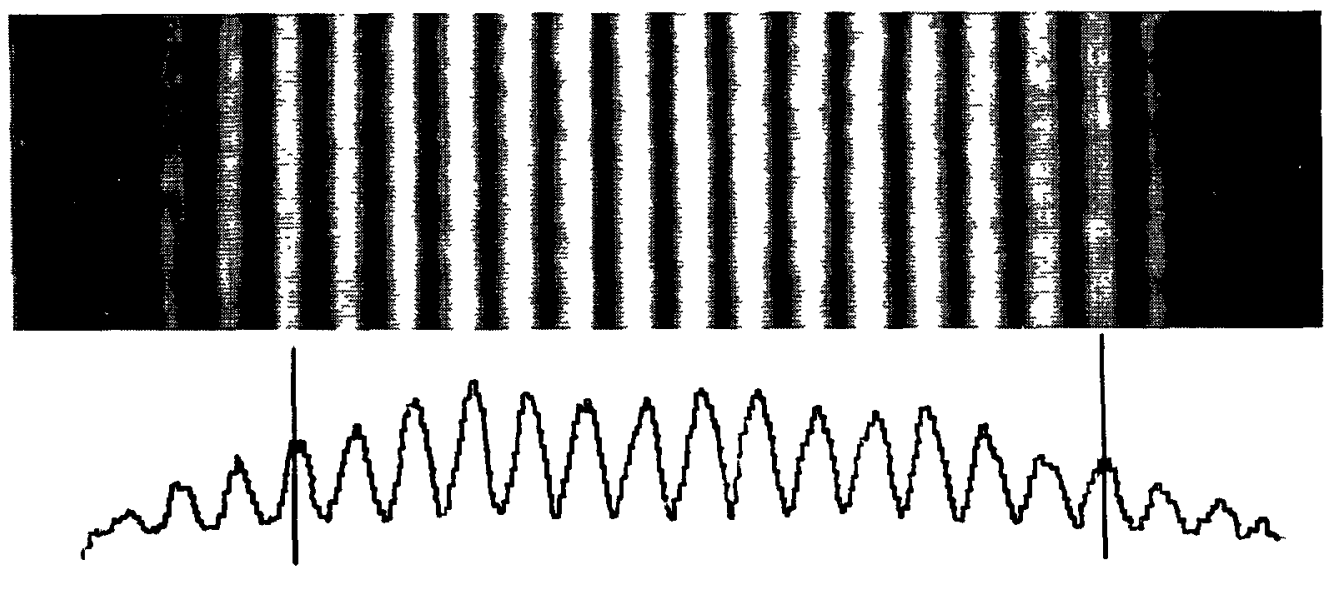

a)

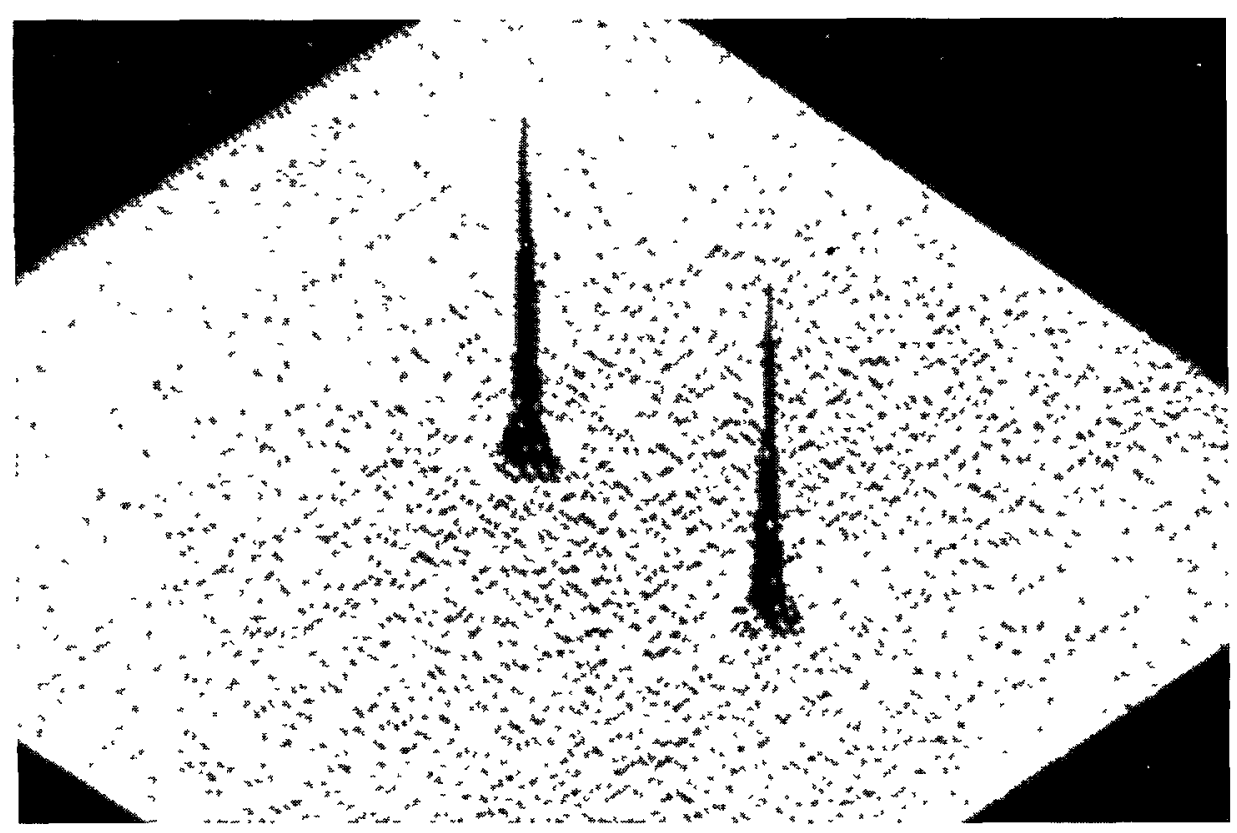

b)

Fig. 6. - a) Illumination pattern in the grid plane. b) Illumination pattern in the far field.

Figure 8 presents the illumination pattern recorded in the square aperture located in the output mirror plane. As we can see, the illumination is nearly constant across the square aperture. The residual fluctuations, of the order of $8 \%$, are due partially to the spectral truncation beyond the grid outline. In order to verify the phase flatness, we examine the illumination in the far field (that is, in the image plane of the grid). The illumination of figure 9 was recorded in the Fourier plane of the square aperture. We can easily recognize a $(\sin X / X)^{2}$ bidimensional function with a main lobe twice as wide as the side lobes. This 


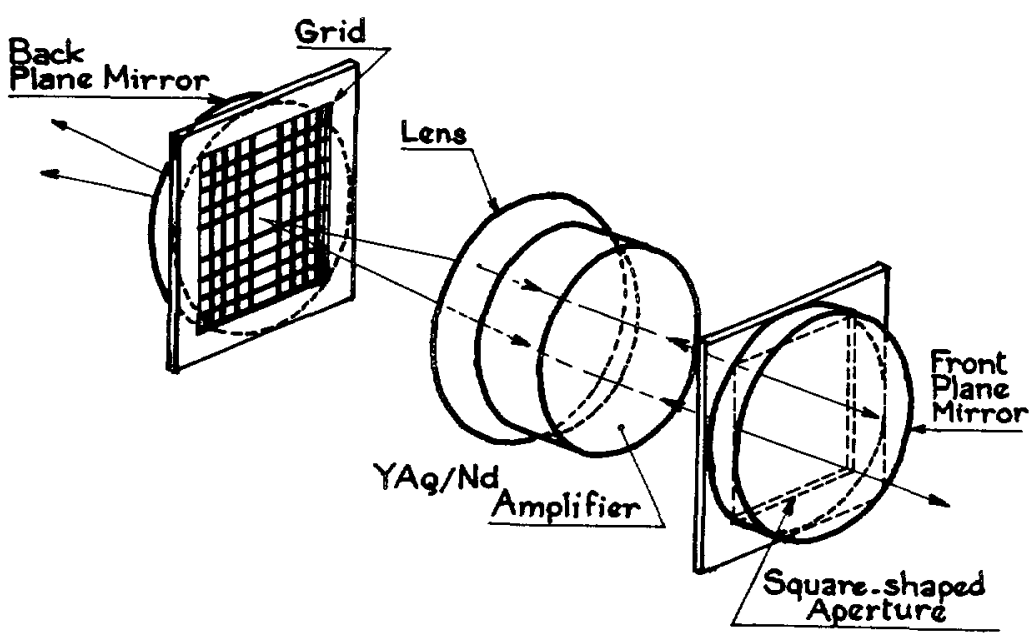

Fig. 7. - Industrial application : synthesis of a square limited PLANE wave.

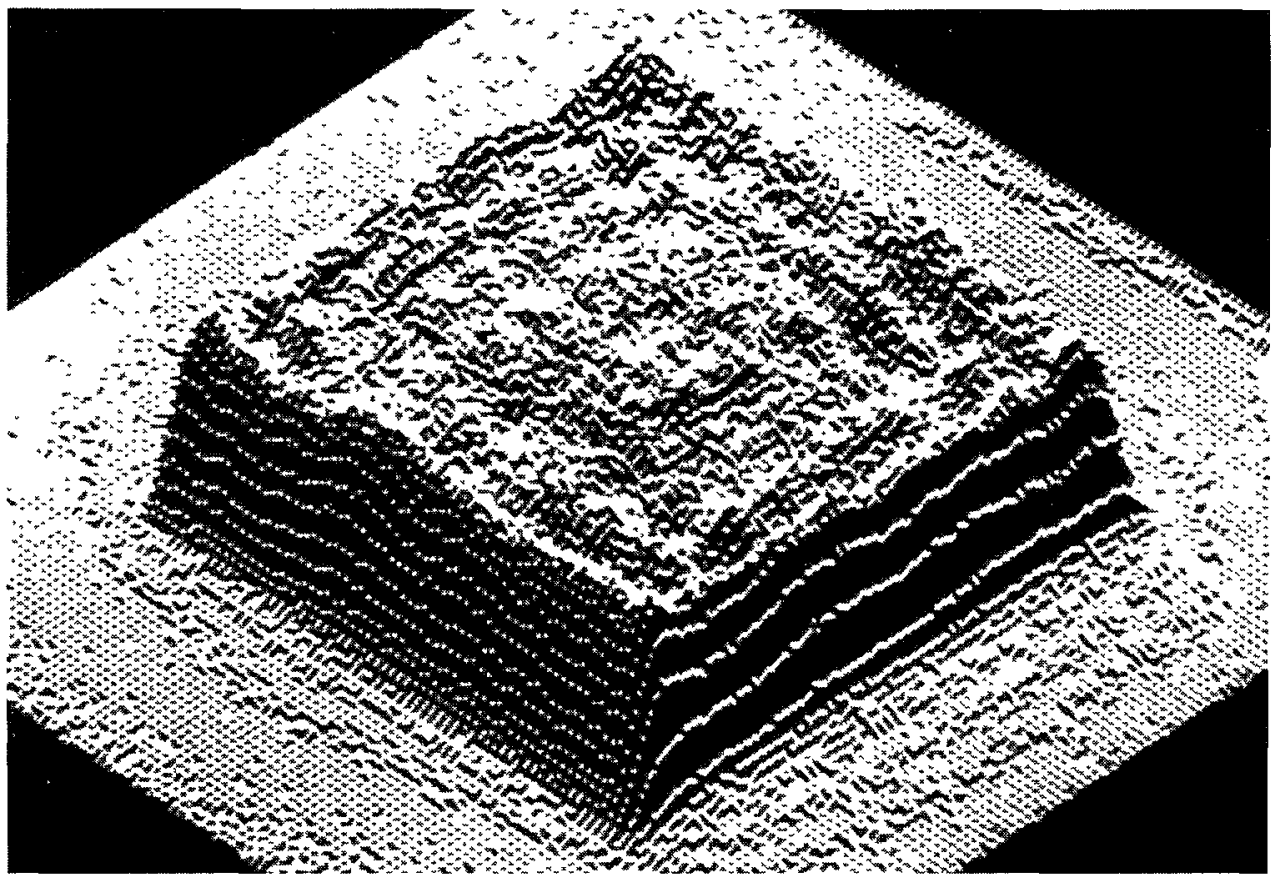

Fig. 8. - Illumination in the square plane aperture.

intensity pattern in fact corresponds to the far field pattern of a plane wave truncated by a square aperture. The number of pixels in the synthesized wave is of the order of 500 .

To control the quality of the reconstructed wave, we have recorded the diffracted patterns in different planes between the square aperture and the far field plane, recognizing the typical Fresnel patterns of a square hole illuminated by a monochromatic plane wave. Then we compared the illuminations in several planes after the output mirror with the illuminations 


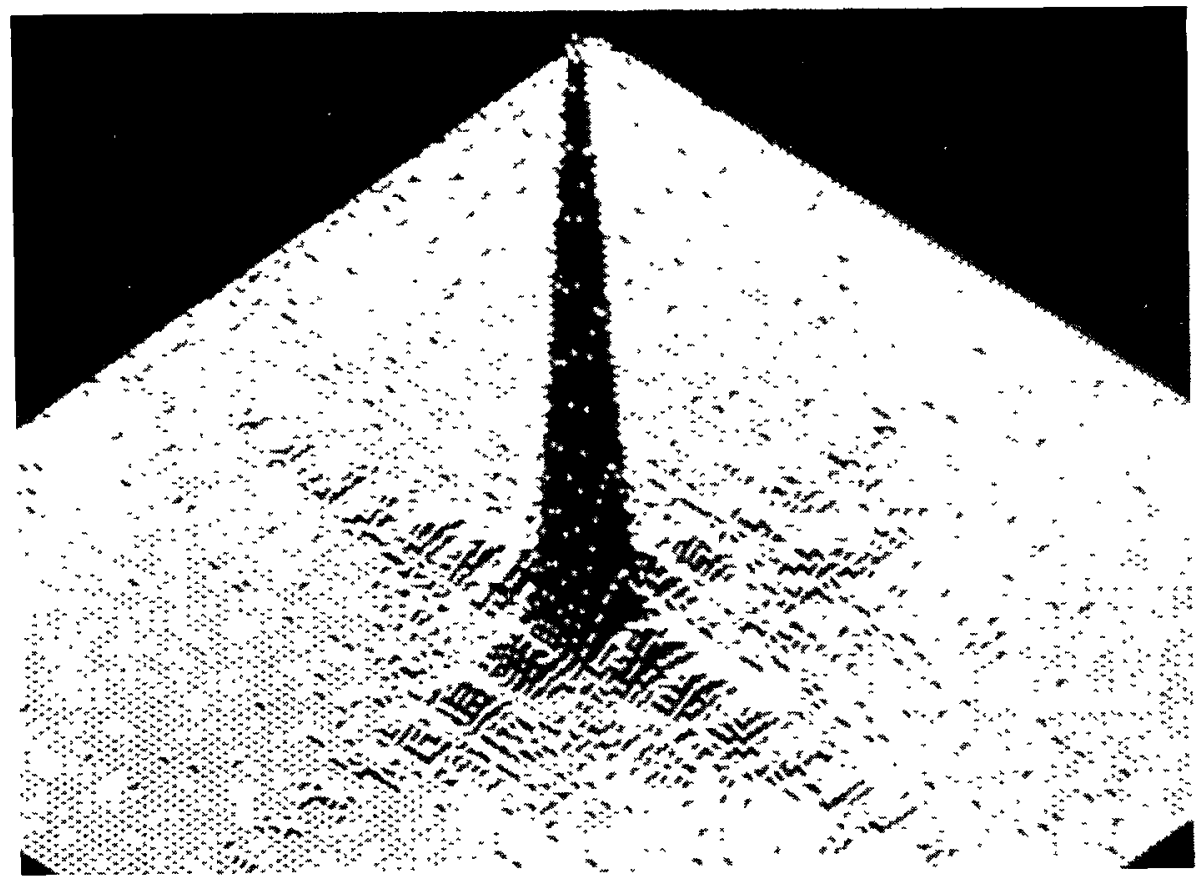

Fig. 9. - Illumination in the far field.

obtained after a square aperture lighted by a He/Ne laser beam (Fig. 10). The wave synthesized by our process, propagates according to the usual diffraction laws, exactly like the wave diffracted by a classical hologram.

The "hologram " which allowed this wave reconstruction is not of the usual type : in the system we propose, it is a pair of binary masks introduced in the confocal laser cavity.
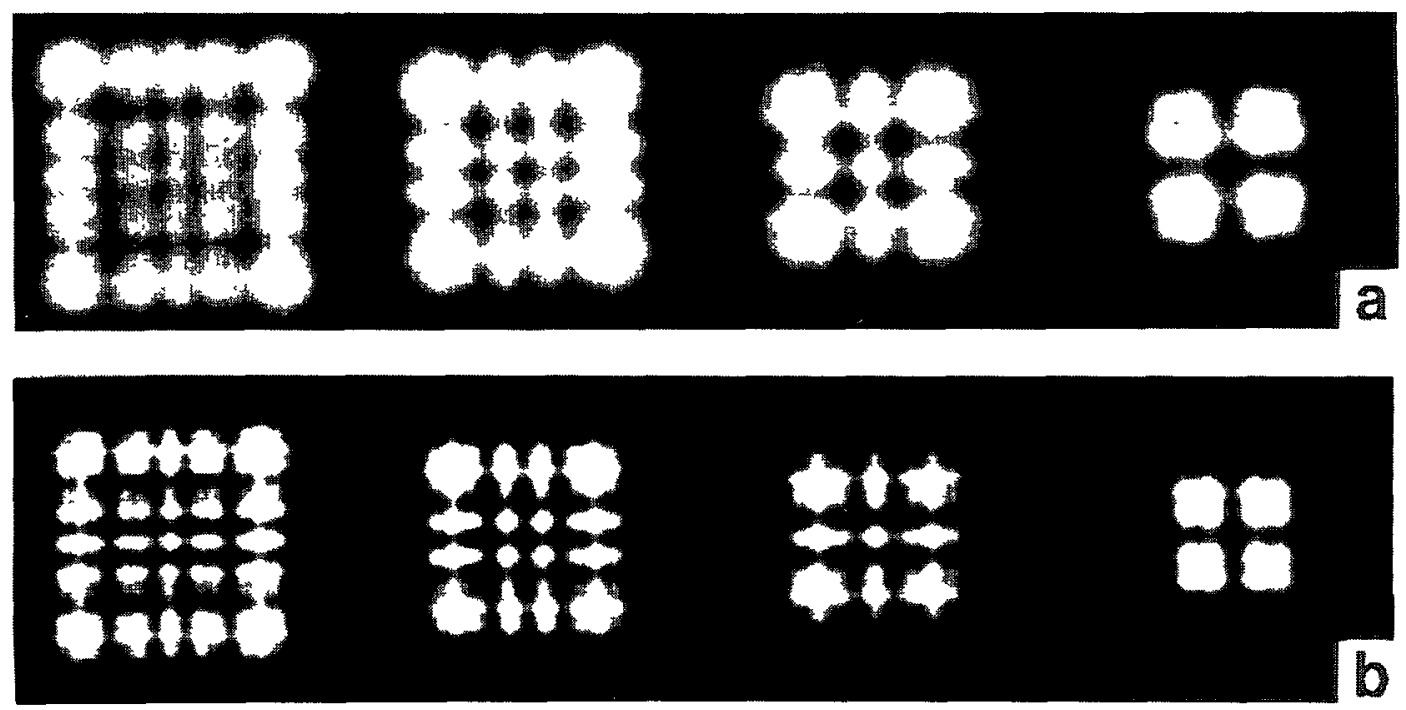

Fig. 10. - Illumination patterns. a) In several planes after the output synthezizer mirror. b) In several planes after a square aperture lighted by a $\mathrm{He}-\mathrm{Ne}$ laser beam. 


\section{Study of the temporal convergence of the process.}

We are also interested in the convergence speed of the process [15]. In other words : how many round-trips have to be performed by light inside the cavity for stable generation of the expected spatial profile?

The experiment reported in figure 11 measures the rise-time of the stable stored field. In this figure, we recognize the synthesizer laser with its two masks, one being a one dimensional slit of width $a$, the other a grid whose black stripes coincide with the minimums of the $\sin (\pi a x / \lambda f) /(\pi a x / \lambda f)$ slit Fourier transform function. In the free-running regime, the first effects of the mask filtering cannot be seen, because the power levels are too weak. For this reason, an additionnal mode laser (L.M.S.) drives the first by a single powerful short pulse of measurable intensity. In the cavity of the resonator, the pulse is amplified and its spatial profile will be reshaped by both masks. After a round-trip, a small fraction of the pulse leaves the resonator, the main part continuing oscillations inside. By this means, we obtain a sequence of measurable pulses with different spatial profiles.

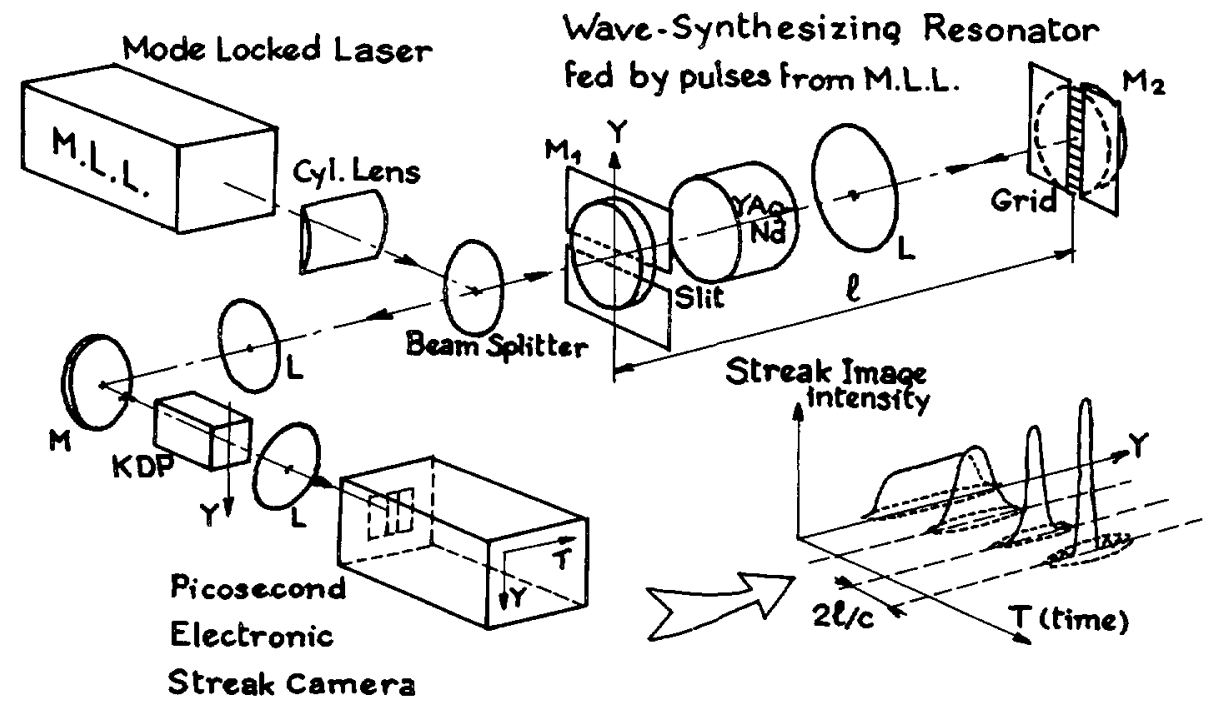

Fig. 11. - Temporal convergence of the process.

A temporal analysis of these one-dimensional profiles (along $Y$ in the figure) with a streak camera makes it possible to observe the convergence process in real time. A frequency doubling crystal (KDP) placed before the streak camera converts the laser light into visible light, because the camera photocathode is not sensitive to the laser infrared radiation. By longitudinally moving the two converging lenses. we can bring different image planes to focus on the streak camera slit. On the graph of figure 11 , the $\mathrm{M}_{2}$ grid is imaged on the slit; the spatial profile converges towards a $\sin (\pi a x / \lambda f) /(\pi a x / \lambda f)$ function.

On the recordings of figure 12 , the vertical axis $Y$ represents the dimension parallel to the slit width and the horizontal one represents time.

i) The slit plane $M_{1}$ is imaged on the streak camera slit. As the total length of time which can be analysed by the camera is about $50 \mathrm{~ns}$ (equal to 7 round-trips) we carried out threee recordings, each lasting approximately $50 \mathrm{~ns}$, in order to observe, with three successive laser 

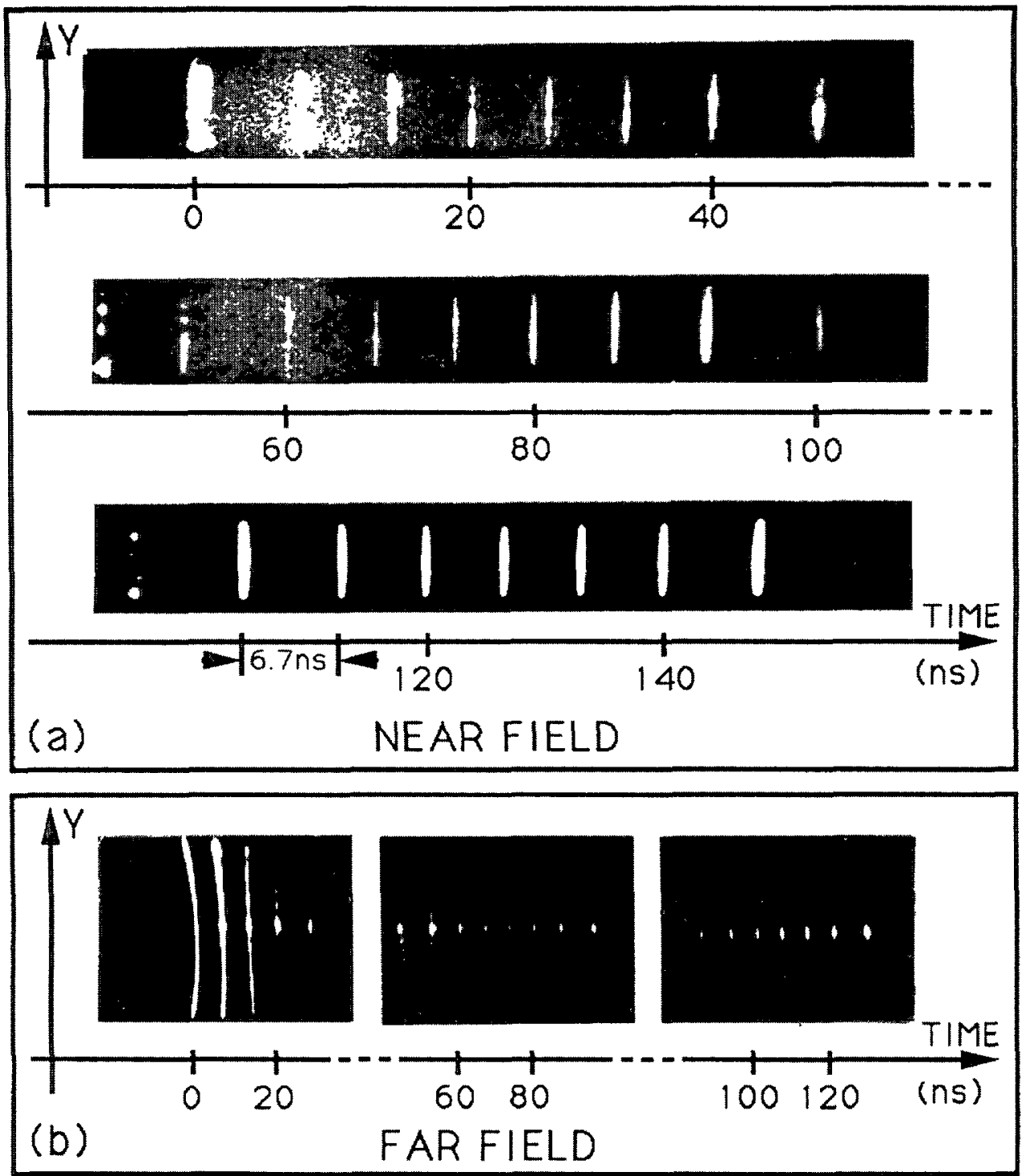

Fig. 12. - For three successive laser shots, recordings of the spatial profiles of the successive pulses, emitted after each round-trip in the cavity : a) in the plane of the output mirror $\mathbf{M}_{1}, b$ ) in the far field.

shots, convergence towards the required profile. The profiles of the first pulses are speckled; the following ones become smoother and smoother; the last ones are almost constant. The few extra white smears between the different profiles come from the noise of the image intensifier.

ii) The grid plane $\mathrm{M}_{2}$ is imaged on the streak camera. We show three successive recordings. The speckle of the first pulses converges quickly towards a $(\sin X / X)^{2}$ function whose central lobe only was recorded, because of the non linear responses of the camera, of the image intensifier and of the film. The narrow dynamic range of the recording process prevents a distinct view of the side lobes of the sinc function. For the last pulses, the filtering imposed by the grid brings about less losses, because the profile is adapted : the main lobe becomes more intense, to the detriment of the secondary lobes. 
In conclusion, the profile convergence is reached after about fifteen round-trips, corresponding to a rise time of the stable pattern of $15 \times 8=120 \mathrm{~ns}$. The process is not instantaneous, differing in that point from usual holography.

\section{Conclusion and perspectives.}

The experiments described in this paper constitute a first step towards the control of the synthesis of non-conventionnal coherent wave profiles inside a laser resonator. We have shown, with simple examples, that it is possible to master the active modal filtering which shapes the beam in any laser oscillator, by using two complementary diffracting screens placed in the resonator. The modes synthesized in this way can be extremely varied and very different from classical ones.

However, it is not always possible to synthesize waves by a pair of masks placed into Fourier planes. Indeed, we may easily find several examples of functions that cannot be generated efficiently by the present process : limited spherical (for instance Gaussian) waves, as they have no zero inside their boundary, or $(\sin X / X)^{n}$ profiles, as they have the same zeroes as $(\sin X / X)$ function.

Among the improvements that we can find to synthesize such waves, we plan to increase the number of masks by selecting planes with a great number of zeroes, or to impose volumic constraints with tridimensionnal filters.

\section{Acknowledgements.}

This work was implemented in collaboration with B. M. Industries Society and supported by the French D.R.E.T. (Direction des Recherches, Etudes et Techniques, Grants n 89125 and 91062) and the E.E.C. (European Economic Community, Value Program).

\section{References}

[1] Barakat R. and Newsam G., Algorithms for reconstruction of partially known bandlimited Fourier transform pairs from noisy data : I The prototypical linear problem, J. Int. Eq 9 (1985) 49-76.

[2] Barakat R. and Newsam G.. Algorithms for reconstruction of partially known bandlimited Fourier transform pairs from noisy data: II The non linear problem of phase retrieval, J. Int. Eq. 9 (1985) $77-125$.

[3] Barakat R. and Newsam G., Algorithms for reconstruction of partially known band-limited Fourier-transform pairs from noisy data, J.O.S.A. A 2 (1985) 2027-2039.

[4] PERI D., Optical implementation of a phase retrieval algorithm. Appl. Opt. 26 (1987) 1782-1785.

[5] LI T. and SMITH P. W.. Mode selection and mode volume enhancement in a gas laser with internal lens, Proc. IEEE (Corresp.) 53 (1965) 399-400.

[6] Smith P. W., Mode selection in lasers, Proc. IEEE 60 (1972) 422-440.

[7] Rigrod W. W., Isolation of axi-symmetrical optical resinator modes, Appl. Phys. Lett. 2(1963) 5153.

[8] D'Amato F. X., Siebert E. T. and Roychoudhuri C., Mode control of an array of AlGaAs lasers using a spatial filter in a Talbot cavity, SPIE Laser Dtode Technol. Appl. 1043 (1989) 100-106.

[9] Siegmann A. E.. Lasers - University Science Books. Chapter 20.3: Gaussien apertures and complex ABCD matrices (1986) pp. 786-792.

[10] Akhmanov S. A., Vorontsov M. A. and Ivanov V. Yu., Large scale transverse nonlınear interactions in laser beams; new types of nonlinear waves : onset of optical turbulence, JETP Lett. 47 (1988) 707-711.

[11] Gerchberg R. W. and Saxton W. O.. A practical algorithm for the determination of phase from image and diffraction plane pictures. Optık 35 (1972) 237-246. 
[12] Vampouille M., Colombeau B., Barthelemy A., Saviot F., Kermene V. and Froehly C., Laser de divergence minimale à section photométrique plate dans le champ proche, Ann. Phys. 16 (1991) 223-224.

[13] Kermene V., Saviot F., Vampolille M., Colombeau B., Froehly C. and Dohnalik T., Flattening of the spatial laser beam profile with low losses and minimal beam divergence, Opt. Lett. 17 (1992) 859-861.

[14] Barthelemy A., Colombeau B., Froehly C., Vampoullle M., Résonateurs optiques à Transformation de Fourier, Patent n 90.04.221.

[15] Kermene V., Vampoullle M., Froehly C., Colombeau B., Temporal laser beam construction under controlled mode filtering. Opt. Commun. 97 (1993) 319-325. 
Classification

Physics Abstracts

$42.30-42.70-42.80$

\title{
Smectic $A$ and $C^{*}$ liquid crystal light valves
}

\author{
L. Dupont, Z. Y. Wu, P. Cambon and J. L. de Bougrenet de la Tocnaye \\ Groupe Optique et Systèmes de Communication, ENST de Bretagne, B.P. 832, 29285 Brest \\ Cedex, France
}

(Received 30 November 1992, accepted 22 Aprl 1993)

\begin{abstract}
Résumé. - Le développement des valves optiques dans le domaıne de l'image et la représentatıon spatiale de l'information a bénéficié des progrès réalisés sur les matériaux «cristaux liquides ». Nous allons étudier tout spécialement les développements récents concernant l'utılisation des phases smectiques $A$ et $C^{*}$. Deux types de valves sont décrites avec leurs modes d'adressage respectifs : le modulateur à adressage optıque : l'information est envoyée par voie optique et la commutation s'effectue par la conversion du signal optique en signal électrique grâce à une couche d'un matériau photoconducteur (silicium amorphe hydrogéné). Le deuxième type de valve est un circuit VLSI recouvert de cristal liquide pouvant être adressé par voie optique et effectuant un traitement logique local de l'information reçue ; la commutation locale du cristal liquide permet alors la lecture de l'état final.
\end{abstract}

\begin{abstract}
The development of light valves for application in the domain of imaging and information display has benefited from simultaneous advances in liquid crystal materials. We will focus on recent developments involving the use of smectic $A$ and smectic $\mathrm{C}^{*}$. Two types of light valves are described with their addressing technology. The first one is optically addressed spatial light modulator : optical information is converted into electric information through the medium of a photoconductivity material layer (amorphous silicon). The second type of valve is a VLSI chip covered with liquid crystal which can be optically addressed on each pixel and perform local electronic processing. The local switching of the liquid crystal allows the reading of the final state.
\end{abstract}

\section{Introduction.}

Optically addressable imaging devices, often referred to as light valves, have played a crucial role in adaptive optics and optical processing during the two last decades. The design of such devices is a direct consequence of the attention payed to real-time coherent optical processing during the seventies, concurrent with the development of coherent laser sources. One of the problems recognized early on was how to interface the coherent optical processors with the external world. The development of light valves for applications in the domain of imaging and information display, has benefitted from simultaneous advances in liquid crystal materials 
such as nematic liquid crystals which asserted themselves very quickly as main candidates for modulating elements in light valve structures.

There are many possible light valve designs, and we do not wish to be exhaustive on the subject. We will instead focus on recent advances in liquid crystal light valves (LCLV), particularly those involving the use of smectic $\mathrm{A}$ and $\mathrm{C}^{*}$ liquid crystal materials. For more exhaustive summaries of light valve structures, the reader may refer to the major contributions [1-5]. We will briefly discuss the current trends in these materials, particularly the various addressing modes that have been proposed. Finally, some examples showing how the devices are used in the field of information processing and neurocomputing will be presented.

\section{Basic principle.}

A light valve is an optical-to-optical image transducer capable of accepting a low intensity, white or monochromatic light input image and converting it, in real time, into an output image with light from another source. Generally the device is designed in such a way so that the input and output light beams do not interact. This gives the device great flexibility in its operating modes (e.g. allowing cascability). Several different structural combinations are possible. In particular, different modulating elements, and addressing layers have been proposed [6, 7]. As previously mentioned, we will only consider the use of liquid crystals as modulating element. The first liquid crystal modulator to be proposed was the nematic liquid crystal modulator.

2.1 LIQUID CRYSTAL MODULATOR DESCRIPTION. - The most commonly used liquid crystals are shaped like tiny rods. Depending on their temperature the rod-like molecules exist in different mesophases between the crystal state. and the completely unordered isotropic state of a liquid. When a liquid crystal is heated to above $100{ }^{\circ} \mathrm{C}$, the molecules are randomly oriented and the solution appears isotropic. As the temperature decreases, the liquid crystal undergoes a phase transition from the isotropic to the nematic phase where molecules have orientational but not positional order. If the temperature is further reduced, the molecules begin to self-assemble into layers (the smectic phase). Therefore, at room temperature liquid crystals are anisotropic due to the ordering of the molecules in the liquid crystalline state $[8,9]$. The easy reorientation of the molecules by externally applied electric fields allows a simple means of electrically controlling the optical properties of the phase.

A liquid crystall cell consists of a sandwich of thin films that electrically control the optical birefringence of a thin $(>2-4 \mu \mathrm{m})$ liquid crystal layer. The general configuration is shown in figure 1. The ac light valve consists of a number of thin film layers sandwiched between two glass substrates. A low voltage (5-10 Vrms) audio frequency power supply is connected to the two outer electrodes (transparent thin film indium-tin oxide (ITO)) and thus across the entire thin film sandwich. The photoconductor and the light blocking layer form a rectifying heterojunction. The dielectric mirror and the blocking layer separate the photoconductor from the readout light beam (Fig. 1). This is one of the major benefits of the ac light valves: it enables simultaneous writing and reading of the device regardless of the spectral composition of the two light beams. A transmissive mode of operation is also possible, in which case the read beam must be chosen out of the absorption band of the photodetector, so that it does not erase the written information. In this case, light passes through the liquid crystal in one direction.

2.2 OpERATING CONDitions. - The twisted nematic light valve used in figure 1 is optically addressed by projection of an incoherent image onto the photoconductive material. The local illumination reduces the photoconductor impedance so that the ac voltage $V$ applied to the cell via the two transparent electrodes is transferred to the electro-optic layer. The molecular 


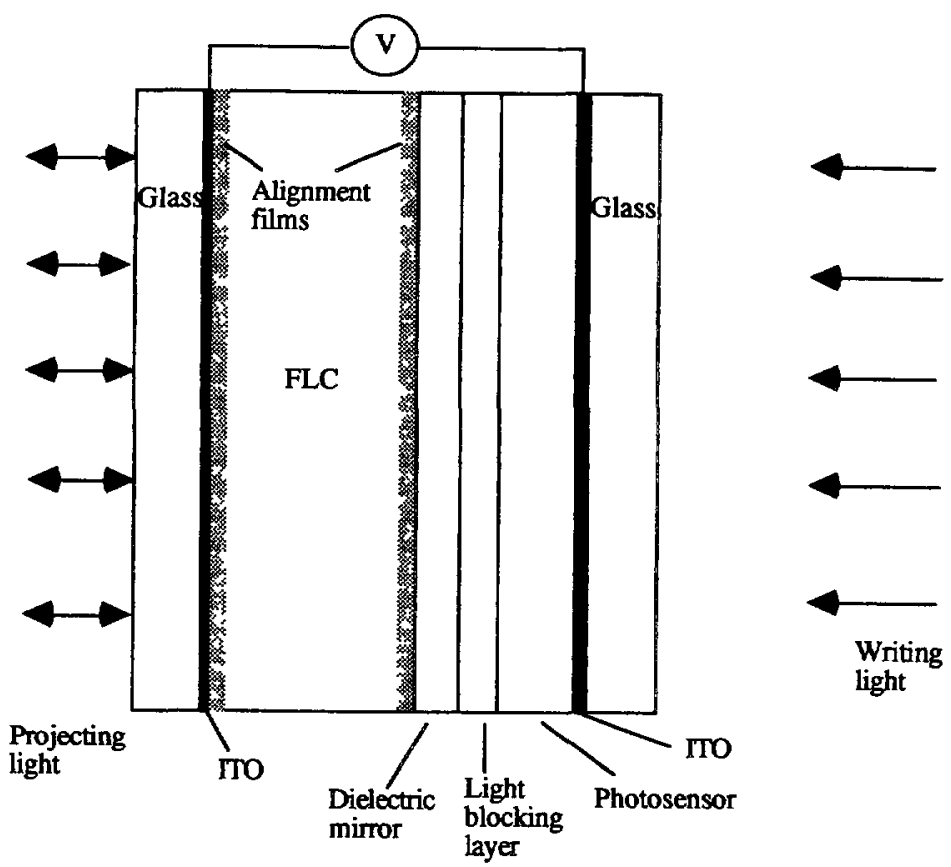

Fig. 1. - Cross-sectional schematıc of the liquid crystal light valve (Ref. [1]).

reorientation induced by the electric field locally changes the liquid crystal birefringence and thus spatially modulates the phase of the incident coherent light beam at wavelength $\lambda$. In this particular mode of recording, the incoherent image is converted into a coherent wavefront having a spatial phase shift $\Delta \varphi$ between two linearly polarized components according to the relation:

$$
\Delta \varphi=2 \pi e \Delta n[I(x, y) ; V] / \lambda
$$

where $e$ is the liquid crystal thickness, $\Delta n[I(x, y) ; V]$ is the photoinduced birefringence change of the liquid crystal due to illumination of the light valve by $I(x, y)$, and $V$ is the bias voltage applied to the transducer. Molecules in the bulk of the liquid crystal layer rotate through the angle of twist between the electrodes. This twisted alignment configuration, combined with the intrinsic optical birefringence of the liquid crystal, causes the polarization direction of a linearly polarized incident light to rotate exactly through the twist angle.

When no electric field is applied, as shown on the right hand side of figure 2 , the orientation of the nematic liquid crystal molecules is twisted a total of $90^{\circ}$ between the two electrodes. In figure 2, we assume that the light input is from the top of the cell. After passing through the polarizer, only the $0^{\circ}$ component of the light continues to propagate. The polarization of the light is rotated a total of a $90^{\circ}$ by the liquid crystal molecules and then blocked by the $0^{\circ}$ polarizer, yielding a dark output which means that the cell is OFF. When the applied electric field is not 0 , as shown on the left-hand side of figure 2 , the molecules are made to tilt towards the direction of the electric field, and in the extreme case can be made perpendicular to the electrodes by applying enough voltage. In this condition, the orientation of the input light is able to pass through the $0^{\circ}$ polarizer, so the output is bright and the cell is $\mathrm{ON}$. When the voltage is below this extreme, the tilt angle of the molecules reduces the rotation angle of polarization of the light passing through the layer. Thus the input-output can be controlled by 
the applied voltage. This means that an amplitude modulation can be obtained when the cell is placed between two crossed polarizers. As simplified expression for the transmittance $T$ has been derived theoretically [10] w.r. to the configuration of figure 2 :

$$
T=T_{0} \sin ^{2}[\pi e \Delta n \phi(V) / \lambda] .
$$

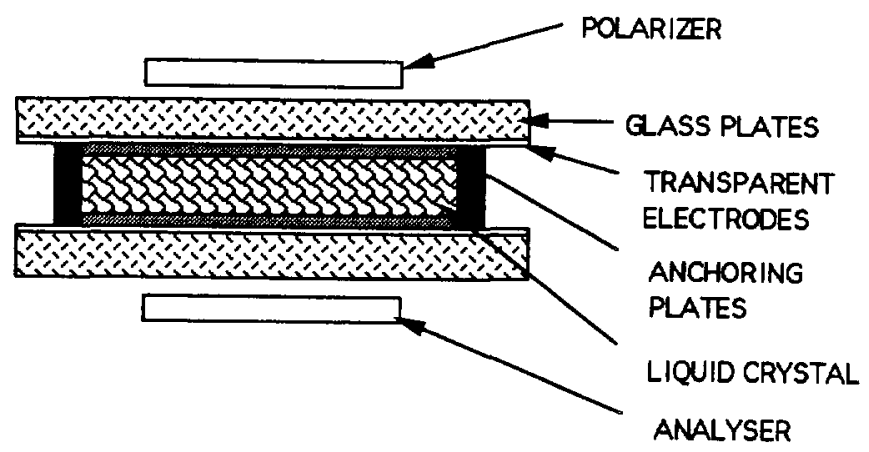

RANDOMLY POLARIZED LIGHT

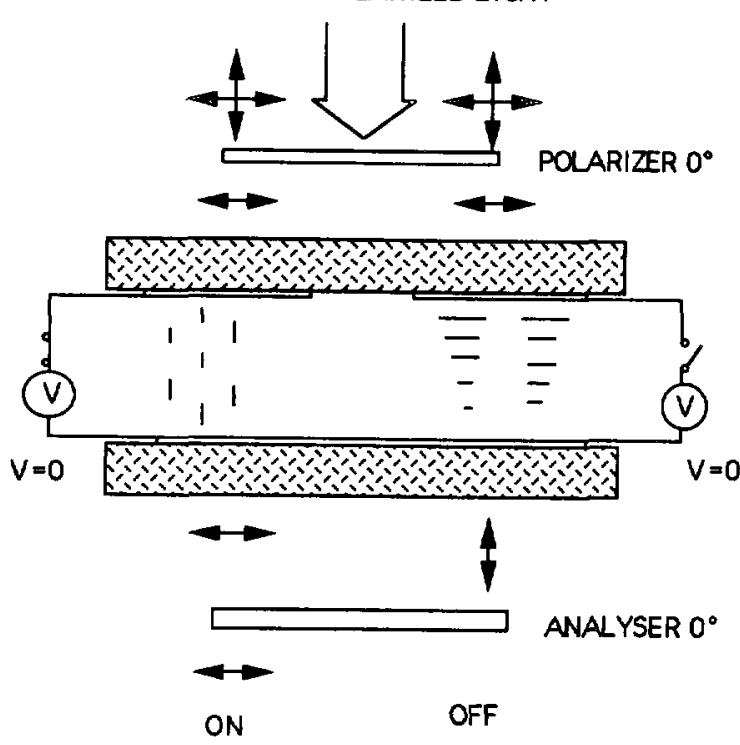

Fig. 2. - Two cases of the light transmission property of the liquid crystal cell.

The transmittance $T$ will thus vary with the voltage $V$, for a given twist angle $\phi \leqslant \pi / 2$. Using this principle, logic operations based on polarization encoding have been proposed [11]. The interesting point here is that an intensity variation is converted into an amplitude variation expressed by the transmittance $T$.

The principle of the twisted nematic mode is based on the fact that the electric torque has a quadratic dependence on the external electric field. This physical behavior implies that the OFF state response time depends on the electric field magnitude, while the ON state relaxation 
time is intrinsic to the twisted nematic [12]. The rise and decay times are given by :

$$
\begin{aligned}
\tau_{\text {decay }} & =\eta / K q^{2} \\
\tau_{\text {rise }} & =\eta /\left|\varepsilon_{\mathrm{a}} E^{2} / 4 \pi-K q^{2}\right|
\end{aligned}
$$

where $\eta$ is the twist viscosity, $\varepsilon_{\mathrm{a}}$ is the dielectric anisotropy, $K$ is the elastic constant function of $E$, and $q=\pi / d$ (where $d$ is the cell thickness).

This relaxation time $\left(\tau_{\text {decay }}\right)$ is the main limitation in the response time for nematic materials. Typical response times of $10 \mathrm{~ms}$ are measured.

\section{Smectic $C^{*}$ and $A$ liquid crystal light valves.}

As seen above, the smectic phase is characterized by layered structures which appear when the temperature decreases. If the long axis of the rod-shaped molecules is perpendicular to the layers, the phase is called smectic A. Lowering the temperature further can cause the molecules to tilt at an angle $\theta$ with respect to the normal of the layer, forming the smectic $\mathrm{C}$ phase, the most ordered and least symmetric liquid crystal phase. A lack of symmetry is needed to make the liquid crystals ferroelectric, that is to possess a spontaneous polarization. Meyer $e t$ al. recognised in 1974 [13] that making the liquid crystals chiral (i.e. unable to be superimposed upon their mirror image by rotation, translation or reflection) removes the liquid crystal mirror plane symmetry thus producing microscopic ferroelectricity.

3.1 Ferroelectric MOde (Goldstone MODE). - Chiral smectic (Sm C*) liquid crystal could have a spontaneous polarization $P_{\mathrm{s}}$. The major advantage of this mode is the high ferroelectric coupling between the external electric field $E$ and $P_{\mathrm{s}}$. The smectic $\mathrm{C}^{*}$ are layered structures where two parameters are needed to describe the physical properties: the spontaneous polarization $P_{\mathrm{s}}$ and the long molecule director axis $n$, which is a function of the tilt angle $\theta$ and the azimutal angle $\phi$ (Fig. 3). Nevertheless, the spontaneous polarization of the material cannot be observed directly because, as a result of the chirality of the phase, a spontaneous helix is formed in which the director axis precesses around the normal of the layers. As a result the polarization is averaged out.

In 1980, Clark and Lagerwall demonstrated the possibility of obtaining a fast switching device with a thin film liquid crystal (called the SSFLC for surface stabilized FLC) where the layers were perpendicular to the electrode [14] (Fig. 4). The basic idea was to prevent the helix formation by a confinement between two glass plates. In the SSFLC structure, due to the strong anchoring of the liquid crystals at the electrode interface and the small thickness of the cell, the helicoidal structure cannot form because the twist and chiral energy become

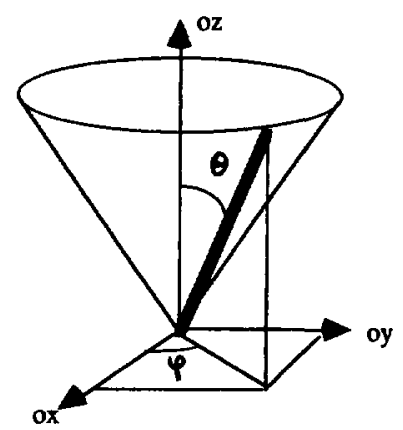

Fig. 3. - Director fluctuations in the $C^{*}$ phase with constant tilt angle (Ref. [13]). 


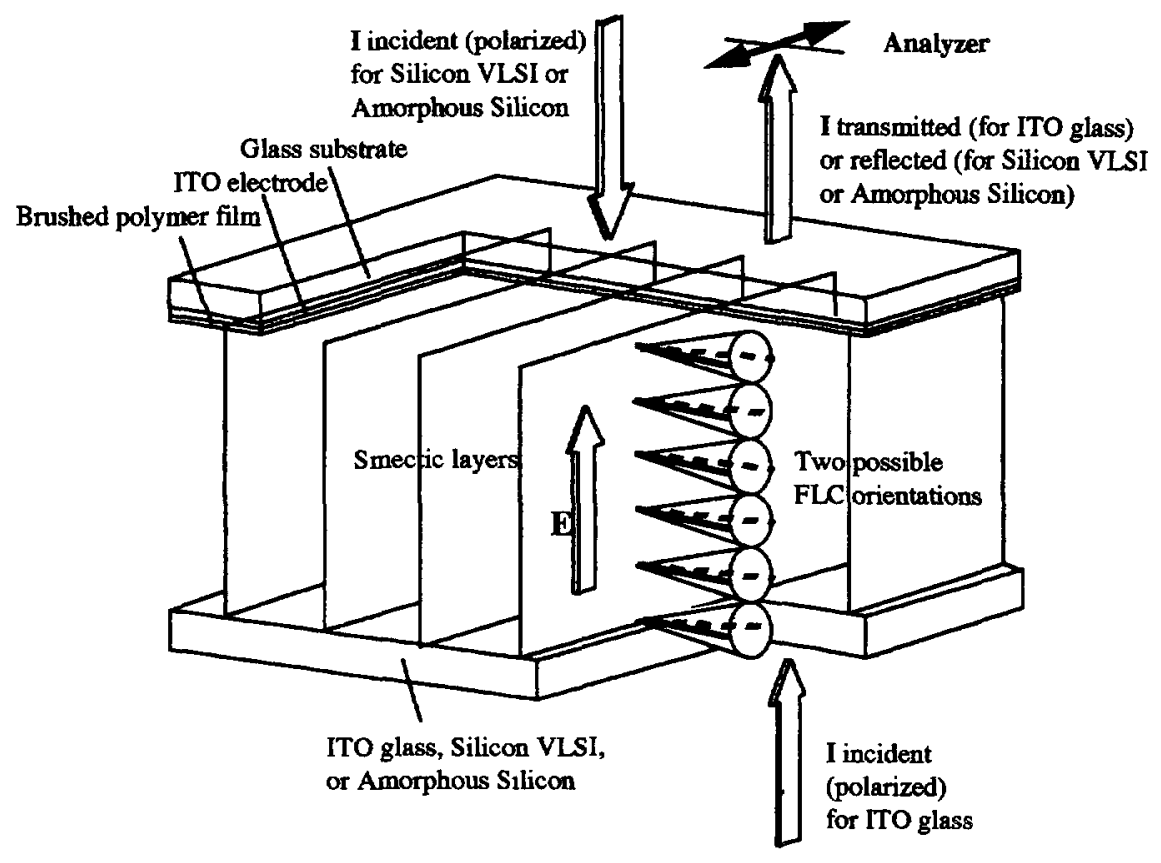

Fig. 4. - Ferroelectric liquid crystal cell configuration.

negligible. In this case, the expression of the Landau free energy $F$ near the smectic A-smectic $C^{*}$ transition is independent of the azimutal angle $\phi$, so $F$ is degenerate on the smectic cone. The SSFLC structure is a twofold degenerated system. In addition to the constraint that the director must lie on the surface of the smectic cone, there is the constraint that it must be parallel to the bounding glass plates. This means that only two positions are possible for $P_{s}$, and the position of $n$ depends only on the direction of the electric field, and on the anchoring of the liquid crystal molecules at the surface. This is the main cause of the bistability.

If the polarization of normally incident light is chosen either parallel or perpendicular to one of these voltage-selected optical axis states, it will be transmitted unaltered. When the other optical axis state is selected by applying a voltage of the opposite sign, the optical axis is rotated to a $\pi / 4$ angle from the incident polarization (in the case of a tilt angle $\theta=22.5^{\circ}$ ) so that both the ordinary and extraordinary modes are excited. Figure 5 illustrates this action of the FLC electro-optic element. The transmission of light through the FLC is thus described by the following equation (Fig. 5) :

$$
T=\sin ^{2}(4 \theta) \sin ^{2}(\pi e \Delta n / \lambda) .
$$

The ferroelectric dynamic mode, in the uniform structure, can be described by the SineGordon equation, if the term for the dielectric coupling and elastic term (gradient term) are neglected :

$$
\gamma_{\phi} \delta \phi / \delta t=-P_{\mathrm{s}} \cdot E \sin \phi
$$

where $\gamma_{\phi}$ is the azimutal viscosity. From (5) we can derive the characteristic electric field dependent response time :

$$
\tau=\gamma_{\phi} / P_{\mathrm{s}} E
$$



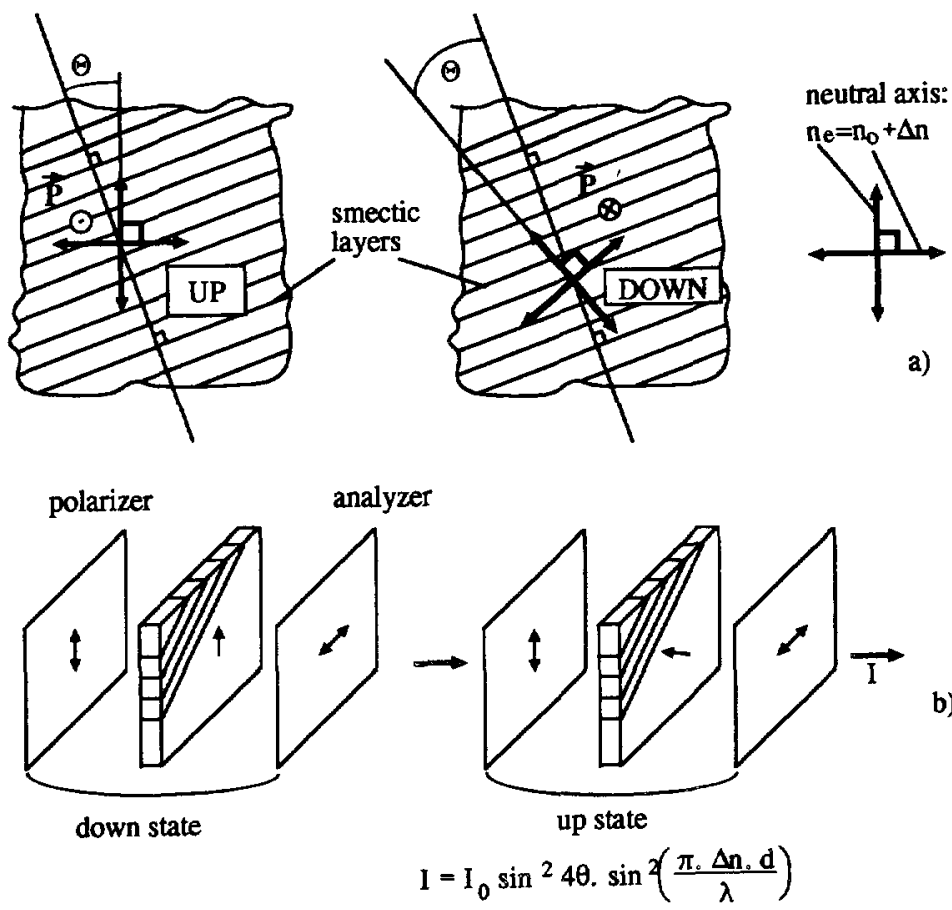

Fig. 5. - Use of a FLC cell as programmable half wave plate.

The difference from the nematic case is thus the symmetry of the response time (i.e. the device can be switched actively in the «ON" and "OFF » states). This is the basis of the faster switching time obtained with the FLC. Typical values of $10-100 \mu$ s or less are measured [14]. This property, together with the intrinsic bistability, are the two original properties of FLC materials.

3.2 ELECTROCLINIC MODE (SOFT-MODE). - In the smectic A phase, a tilt angle can be induced by an applied field $E$ [15]. The structure is the same as the SSFLC, but the alignment of the liquid crystal is better than in the smectic $C^{*}$ (no chevron structures). The existence of this electroclinic mode is due to the coupling between $\theta, P$ (the induced electroclinic polarization) and $E$ in the phenomenological Landau free energy expansion :

$$
F=\frac{1}{2} \alpha\left(T-T_{\mathrm{c}}\right) \theta^{2}+\frac{1}{2 \varepsilon_{0} \chi_{\perp}} P^{2}-c P \theta-P E+\cdot
$$

where $c$ is the linear coupling between $P$ and $\theta, \alpha\left(T-T_{\mathrm{c}}\right)$ is the elastic modulus which depends linearly on the temperature in a first approximation. $\chi_{\perp}$ is a generalized susceptibility.

At equilibrium we obtain the relations :

$$
\frac{\partial F}{\partial \theta}=\frac{\partial F}{\partial P}=0 \Rightarrow\left\{\begin{array}{l}
\theta=\frac{\chi_{\perp} c}{a^{*}} E \\
P=\frac{\chi_{\perp}^{2} c^{2}}{a^{*}} E
\end{array} \text { with } a^{*}=\alpha\left(T-T_{\mathrm{c}}^{*}\right)\right.
$$


$T_{c}^{*}$ is the renormalized transition $\mathrm{SmC}^{*}$-SmA temperature for chiral compound. This means that there is a linear relation between $\theta, P$ and $E$. This linear relation is crucial because it is the basis of the formation of grey scales. The dynamic equation is given, in this case, by the following Landau-Khalatnikov relations :

$$
\begin{aligned}
\gamma_{\theta} \frac{\partial \theta}{\partial t} & =-\frac{\partial F}{\partial \theta} \\
0 & =\frac{\partial F}{\partial P} .
\end{aligned}
$$

Where $\gamma_{\theta}$ is the soft-mode viscosity. The dynamics of the soft mode looks like a Lorentzian relaxation, from which we reduce the characteristic response time of the electroclinic mode :

$$
\tau_{\text {elec }}=\gamma_{\theta} / a^{*}
$$

which is independent of the external electric field value. This mode has a critical temperature behaviour near the SmA-SmC* transition. The divergence of the response time follows the law :

$$
\tau_{\mathrm{elec}} \approx \frac{1}{\left(T-T_{\mathrm{c}}^{*}\right)}
$$

In the soft mode, the highest contrast is associated with the smallest speed : a compromise must be found. This implies good thermal regulation to perform the dynamics.

Typical switching of 1-10 $\mathrm{s}$ can be observed, making the soft mode the fastest liquid crystal electrooptic effect [15].

3.3 BISTABILITY AND GREY LEVEL ON SMECTIC $C^{*}$. - Although SSFLCs are basically bistable devices, it is possible to obtain grey levels under certain driving conditions [16] (e.g. for an under critical value of the product of height and length of the applied voltage). Under these conditions, the cell does not switch uniformly, but shows a grainy structure due to the appearance of small black and white domains. Averaging the optical response over a given area results in a grey level. One advantage of creating grey levels with bistable devices instead of other materials like nematics or smectic A is the memory of the device. The grey level can be stored, and in the case of an optically addressed spatial light modulator (OASLM), optically read and modified. This is a very powerful property for a large number of applications in optical computing and display [17].

The grey level operation is due to multi-domain switching, as described in [18]. Switching by wall propagation is less energy consuming than uniform switching. The spatial distribution of switched domains is determined by the more or less random distribution of defects in the smectic layers structure which act as nucleation sites for the domains. Another interesting property is the linearity of the process. It has been shown [16] that the grey level process is linear when charge controlled and strongly nonlinear when voltage controlled as when used with photoconductor addressing.

3.4 THE PERformanCE OF LiQuid CRYSTAL Light VAlves. - The performance of the liquid crystal light valve for optical data processing applications is measured by the following parameters : contrast, resolution, linearity, response time, optical flatness and image quality. Contrast. - This feature is very critical because it allows, together with the linearity, possible information enhancements in cascaded architectures. The contrast is the maximum ratio of the polarized light intensity passing through the valve at state ON to that at state OFF. In the case of nematic materials, it is mainly limited by the voltage value necessary to tilt the molecules. It 
can be altered principally by liquid crystal defects. This is the case for FLC materials where the so-called zig-zag [19] or other kind of defects can appear if appropriate alignment techniques are not used. Contrast value ranging from 20-500:1 can be typically expected with standard FLC OASLMs [20]. At Boulder contrast ratios that exceed 1000:1 for a laser beam of $1 \mathrm{~mm}$ diameter have been measured for individual optical shutters [20].

Linearity. - For most modulators, the contrast is not a linear function of the writing beam intensity. Firstly, they are used in conjunction with crossed-polarizers which produce an output intensity proportional to the square of the sine of the input intensity. Secondly, for the photoaddressing layer its photoconductivity is a non-linear function of the illumination $[21,22]$. The variation of the photoconductivity can be described as :

$$
\sigma_{\mathrm{ph}}=\sigma_{0} F^{\gamma}
$$

where $\sigma_{0}$ is a prefactor and $F$ is the illumination intensity. For amorphous silicon, when $F$ is in the order of 0.1 to $10 \mu \mathrm{W} / \mathrm{cm}^{2}, \gamma>1$ and the photo-conductor operates in the so-called super linear region; in most cases $F=10 \mu \mathrm{W} / \mathrm{cm}^{2}-10 \mathrm{~mW} / \mathrm{cm}^{2}, \gamma=0.7-0.9[23,24]$; if $F>10 \mathrm{~mW} / \mathrm{cm}^{2}, \gamma$ is about 0.5 . The voltage applied to the liquid crystal is the difference between the valve total voltage and the voltage applied to the photo-addressing layer. When amorphous silicon is illuminated, the voltage applied to the liquid crystal increases. Naturally the voltage, and therefore the contrast, vary non-linearly with the intensity of the writing beam (see Fig. 6). Initially the contrast increases nearly linearly with the writing beam intensity, after that, it reaches saturation, where the OASLM current is limited by the FLC resistance and almost all of the valve voltage is applied to the liquid crystal. It should be mentioned that because of the dependence of photoconductivity on the Fermi level position, the semiconductor/contact interface may change the slope of the arising part of the contrast and the repartition

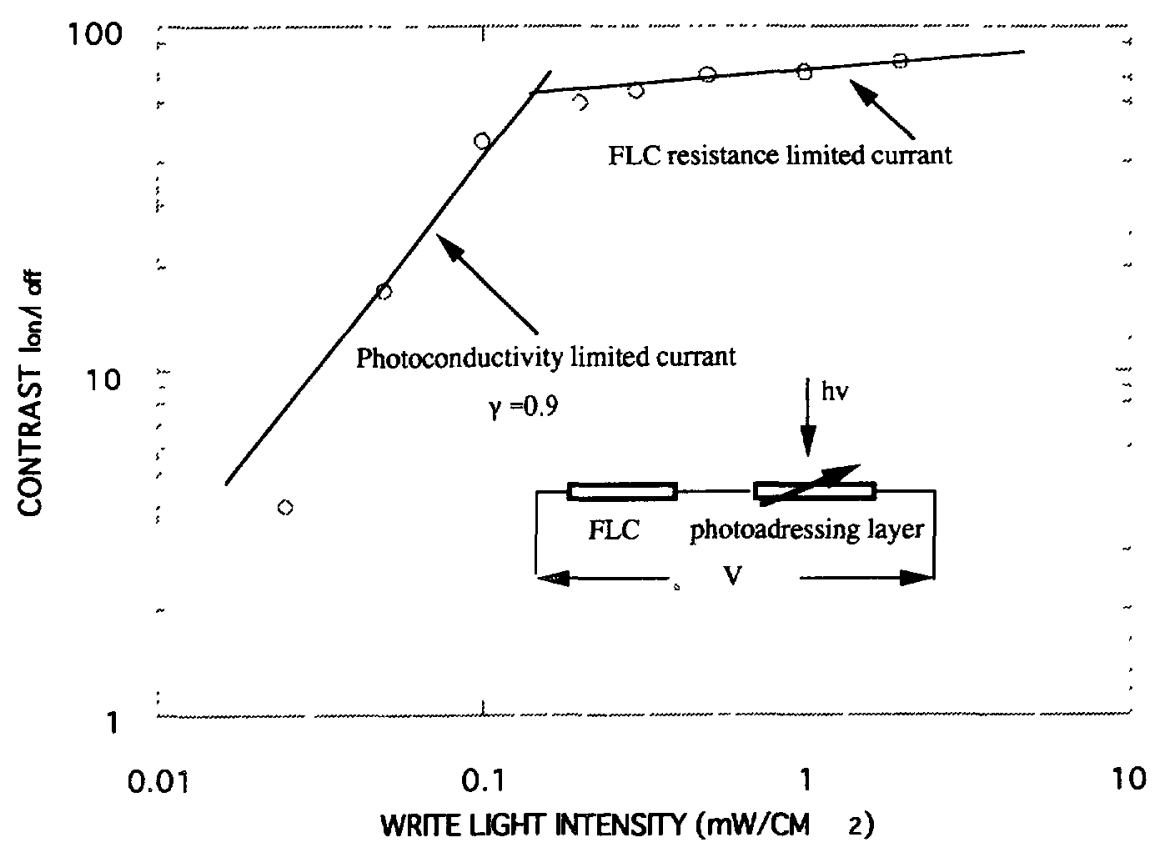

Fig. 6. - The non-linear behaviour of the contrast versus the intensity of writing beam. 
of the voltage between liquid crystal and photon-addressing layer may shift the transition of contrast saturation.

Another important category of SLM nonlinear operations is image thresholding. To start writing, every valve has a minimum voltage applied to the liquid crystal. In the OASLM case, there is a writing beam intensity which corresponds to the minimum voltage. As explained above, this threshold writing intensity is determined by the photoconductivity behavior and the repartition of the voltage between the photo-addressing layer and the liquid crystal and therefore can be tailored according to the choice of materials and the design of this modulator.

Resolution: (given at 50\% MTF). - It is a critical feature for 2D processing because it determines the parallel processing capacity of the device. In the case of light valves, two features have to be considered : the photo-addressing layer and the liquid crystal itself. As far as the photo-addressing layer is concerned, to have a good resolution one has to prevent transversal leakage of charges from illuminated lines to its nearest dark neighbours, in other words, the resolution is dependent on the square resistance and the thickness of the photoaddressing layer. Obviously, the higher the resistance and the thinner the layer, the better the resolution will be. If the photo-addressing layer is compensated amorphous silicon of $10^{-12}(\mathrm{ohm}-\mathrm{cm})^{-1}$ and $5000 \AA$ thickness, the resolution may be as high as $150-180 \mathrm{pl} / \mathrm{mm}$. Concerning the liquid crystal, its conductivity is of the order of $10^{-9} 10^{-10}(\mathrm{ohm}-\mathrm{cm})^{-1}$; the thickness is about $2 \mu \mathrm{m}$, the resolution is limited by the domain size, in the case of FLC. The role of other layers such as dielectric mirror and alignment coatings can be neglected. A spatial resolution of $40 \mathrm{lp} / \mathrm{mm}$ at $50 \% \mathrm{MTH}$ has been measured for the FLC OASLM described in [20] and [21], while a resolution of 125 line pairs per $\mathrm{mm}$ is generally given in the case of a FLC SLM or display.

Response time. - It is of particular interest, for example, when the device is optically addressed. Typical switching times of 10-100 $\mu$ s can be obtained with smectic $C^{*}$ FLC and switching times of 1-10 $\mu$ s with smectic A. This is related (Eqs. (6) and (9)) to the value of $P$ and $\gamma_{\theta}$. Experimental mixtures have achieved $P>500 \mathrm{nC} \mathrm{cm}^{-2}$ These improvements would result in microsecond switching speeds at $25^{\circ} \mathrm{C}$, and $120 \mathrm{~ns}$ at higher temperatures. Concerning the viscosity $\gamma_{\theta}$, the energy dissipated by the viscous motion of the molecular director is likely to limit the switching speed. A typical value for FLC material viscosity in a room-temperature device is $50 \mathrm{cP}$. For a reflection mode device of thickness $1 \mu \mathrm{m}$, if the device can dissipate $10 \mathrm{Wcm}^{-2}$, switching speeds of $1.3 \mu \mathrm{s}$ at room-temperature and $130 \mathrm{~ns}$ at elevated temperature $\left(\gamma_{\theta}=5 \mathrm{cP}\right)$ are possible [25].

Nevertheless in the case of light valves, the response time is related to the structure. In a photoconductor configuration, the response time may be rather long. Because in microcrystalline or amorphous semiconductors, carriers are captured and emitted by shallows traps during the transport. the transport being a dispersive process [26]. A photoconductivity decay is thus formed. The response time depends on the trap density. If one uses a photodiode structure as the photo-addressing layer, a built-in field controls the whole thickness of the layer, carriers are collected more easily. The response time decreases considerably. This is the reason why photodiode or phototransistor structures are preferable with FLC.

Finally, optical flatness (measured with an interferometer) and the image quality should not be neglected. In practice, this involves principally technological excellence. Furthermore, a coherent optical SLM is naturally more demanding in flatness, so that sophisticated planarization is required. 


\section{The main addressing modes.}

As mentioned previously, a light valve is a combination of two elements : the addressing layer and the light modulator. The addressing layer is critical not only because it determines the operating functionality of the complete device, but also because it will influence directly the electrical and physical behaviours of the complete device. This point should not be underestimated when assigning to the device its characteristics. In practice, this imposes compromises between speed, sensitivity, absorption, resolution, etc. Furthermore, external constraints resulting from the system in which the device is inserted, have to be considered (operating wavelength, cascadability, etc.). Basically, there are two main addressing modes. The massive one (using the bulk crystal) and the pixelated one. The latter can be divided into three categories : electrical addressing, optical addressing and smart addressing.

4.1 The Electrical AdDressing. - There are three methods for electrically addressing an FLC SLM : direct drive, matrix and silicon backplane addressing. In direct drive addressing, each pixel in an array has a separate electrode. In matrix addressing. transparent row and columm electrodes are etched onto separate glass substrates. The pixel is defined as an overlap of a row and a column electrode. Only $2 N$ electrodes are required to switch $N^{2}$ devices. The silicon backplane uses a parallel addressing scheme, which is detailed in [27]. The fabrication of liquid crystals on top a silicon integrated circuitry was pioneered by Vass at Edinburgh University, Crossland at BNR Europe and commercial prototypes have been produced by Toshiba, Hughes Aircraft and STC Technology [27]. The first example of a FLC/silicon backplane display used a DRAM addressing of $240 \times 240$ pixels on a $60 \mathrm{~mm} \times 60 \mathrm{~mm}$ die. It was designed as a handheld data display [27]. This addressing scheme does not belong properly do the standard category of light valves, it concerns the category of digital to optical converter. Nevertheless, it is interesting to mention it because such a device was the basis of the recent advances in the domain of smart light valves and retina (see Sect. 4.3).

4.2 THE PHOTO-ADDRESSING. - This configuration corresponds properly to what is commonly called a light valve. In an optically addressed SLM (OASLM), a write beam carrying spatially varying information, is absorbed by the photosensor and the SLM modulates either the phase. polarization, intensity or wavelength of a read-beam. The information can be stored using either slides or CRT. Several groups have produced OASLMs coupling hydrogenated amorphous silicon [28], or GaAs [29] photosensors with FLC modulators. As a photosensor, the a-Si:H has been shown to have many advantages over crystalline photosensors. It has an excellent ratio of photoconductivity $\left(10^{-4}(\mathrm{ohm}-\mathrm{cm})^{-1}\right.$ under $100 \mathrm{~mW} / \mathrm{cm}^{2}$ illumination) over its dark conductivity (say, $10^{-9}(\mathrm{ohm}-\mathrm{cm})^{-1}$ for the undoped : $10^{-12}(\mathrm{ohm}-\mathrm{cm})^{-1}$ for the compensated) according to which OASLM can have a good signal/noise. Amorphous silicon has also a coefficient of absorption of an order of $10^{4}-10^{5} \mathrm{~cm}^{-1}$ at visible region. A photo-addressing layer of just several thousands angstroms is thick enough. This thickness allows us to obtain a high resolution. And lastly, the plasma CVD technique can now be used to deposit hydrogenated amorphous silicon at a low temperature to form a layer with an uniformity as good as $1-2 \%$ over a substrate of $30 \mathrm{~cm} \times 30 \mathrm{~cm}$.

When using a truly bistable OASLM, a light beam can operate either in a reflective or in a transmissive mode. In the transmissive mode, a compromise between the quantum efficiency (i.e. the write sensitivity) and the transparency or absorption has to be found. In practice, this purpose supposes the deposition of thin semiconductor layers. Fortunately, this acts in favour of high resolution. Concerning the device speed, it is limited by the photoconductor. This is generally not a limitation when using a nematic OASLM, because the switching speeds are comparable to that of the relaxation time. However, when using FLC materials, the 
photodetector response speed could become a limiting factor. Such a consideration has given rise to original contributions in the field of the design of fast speed light valves. Moddel was the first to propose associating a bistable FLC with an amorphous silicon p-i-n structure [30]. We have ourselves proposed a $p-i-n-i-p$ or $n-i-p-i-n$ symmetric structures (Fig. 7). These structures give a symmetric photo $I-V$ characteristics which are compatible to the bistability of FLC. Furthermore, they have a good time response. Experiments carried out on such a device have shown repetition rates faster than $20 \mathrm{kHz}$ for an addressing light power of about $25 \mathrm{~mW} / \mathrm{cm}^{2}[31]$.

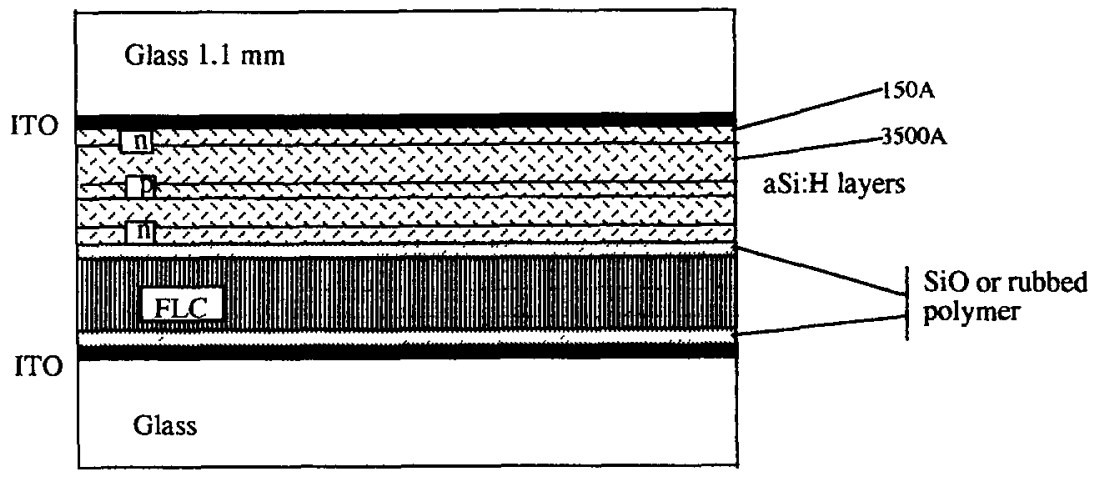

Fig. 7. - Light valve using n-i-p-i-n a-Si:H structure with bistable FLC.

4.3 THE SMART ADDRESSING. - This device can still be considered as a light valve, because of the optical to optical conversion, although this conversion is performed separately on each pixel. This means that each pixel operates independently as a small light valve. Furthermore, in this case, the conversion operation is more complex because intermediate electronic processings can be included during the process (e.g. nonlinear processings [32]) as well as the interaction with the neighbours. This is a reason why the device is called a smart light valve. Smart OASLMs have been fabricated using silicon VLSI backplanes [32, 33]. Figure 8 shows the principle of a such device which combines silicon junction phototransistor as detectors. silicon CMOS analog circuitry, and FLC binary light modulator cells driven directly by the circuitry. Such devices have been proposed to perform, for example, $(32 \times 32)$ image thresholding functions. Switching energies of FLC materials are typically a few $\mathrm{fJ} / \mathrm{mm}^{2}$, and switching speeds in tens of microseconds can be contemplated for binary FLC modulators driven from a silicon VLSI die. Analog operation at comparable switching energies and speeds is expected from smectic A materials. Although CMOS logic speeds are much higher than FLC switching rates, the low static power dissipation of silicon CMOS logic permits a subpicoJoule speed-power product to prevail at low clock rates. This reciprocity allows CMOS circuitry to operate at rates matching liquid crystal switching times $\left(10^{3}-10^{5} \mathrm{~Hz}\right)$ without suffering an increase in switching energy.

Regarding the possibilities of VLSI circuits, more complex designs may be addressed. For example, each pixel can be coupled to a local programmable processor or interconnected with other pixels following a given neighbourhood function. Binary Mathematical Morphology retina [34], or cellular automata [35], have been implemented or studied following this principle. Here, in comparison with the above mentioned devices [32, 33], each cell has been interconnected with other cells, therefore allowing a considerable system complexity, 

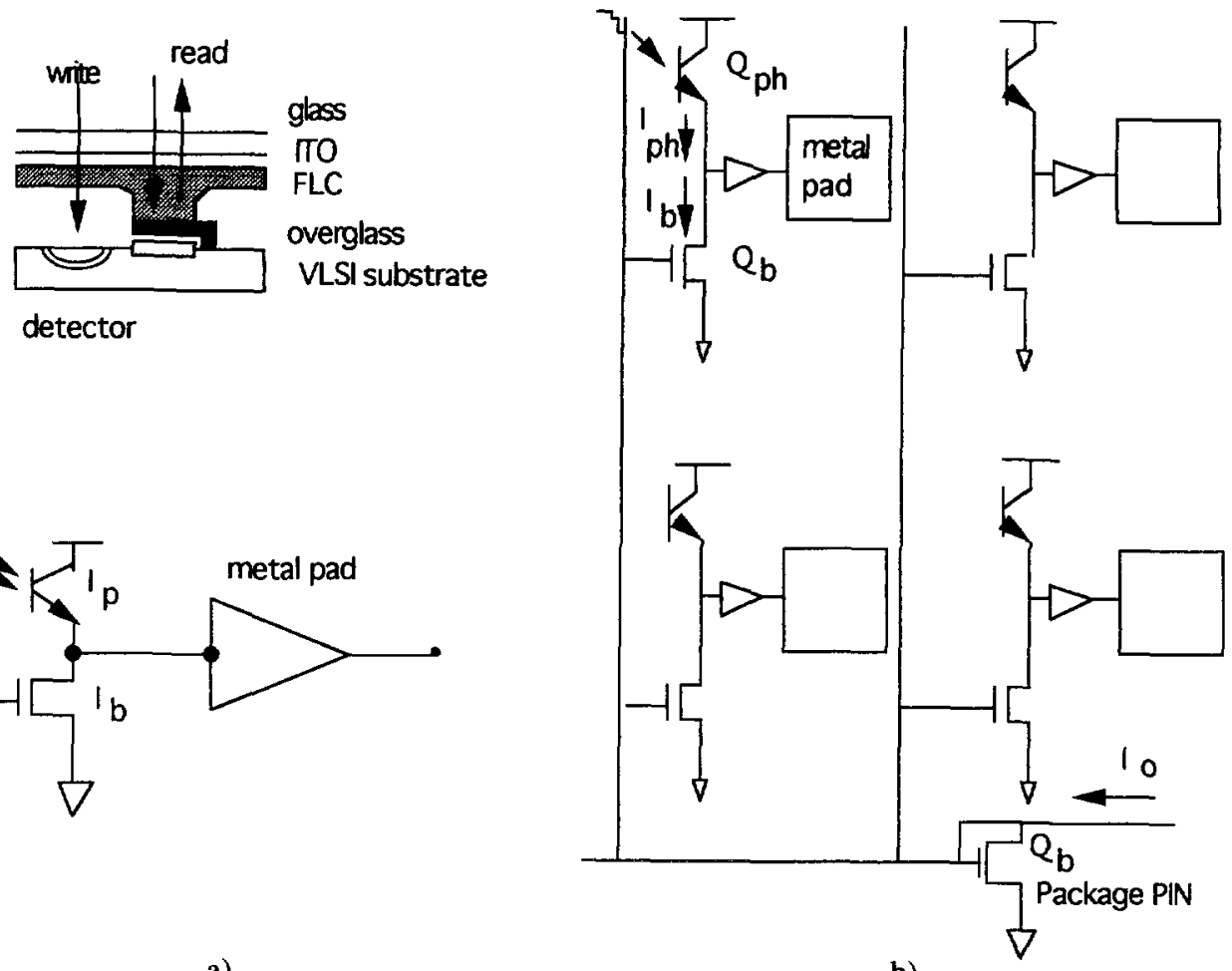

a)

b)

Fig. 8. - Principle of the photoaddressable silicon VLSI/FLC light valve. a) cross section of the VLSIO and functional circuitry, b) schematic circuit diagram of thresholding array (Ref. [26]).

involving, for instance, the implementation of local image processing or cellular automata. In such smart light valves, cell interconnections can be carried out either electronically or optically. For local interconnections (e.g. the nearest neighbourhood) electronical solutions are preferable. This is the case for the retina [34] on which FLC has been deposited successfully [36]. Figure 9 shows a photograph of several cells on the present chip. This chip supplied by the Institut d'Electronique Fondamentale in Orsay, is a silicon chip using $2 \mu \mathrm{m}$ CMOS technology. Each elementary cell is $95 \mu \mathrm{m} \times 95 \mu \mathrm{m}$, and the retina is a matrix of $64 \times 64$ elementary cells, yielding an overall size of $7.2 \mathrm{~mm} \times 7.2 \mathrm{~mm}$. Each cell consists of a photodiode, two memory points, and a transistor coupled logic (TLC) circuit. The photodiodes are silicon photodiodes having a size of $30 \mu \mathrm{m} \times 30 \mu \mathrm{m}$. The TLC circuit allows programming of the shifts (up, down, left, right), and assures the interconnections between the pixels. A glass plate with the transparent counter-electrode has been placed on the chip, and the space between the two parts has been filled with smectic $C^{*}$ liquid crystal (Fig. 9).

During operation, the component is illuminated with an image. Photodectors binarize the image using a pre-set threshold, and memorize it. The exposure time is determined by an average intensity. Each mirror receives a voltage corresponding to the binary value of the pixel, and the voltage difference between the mirror and the counter-electrode determines whether or not the liquid crystal above the mirror will switch. During reading, a uniform and linearly polarized incident light beam is reflected by the mirrors and modulated by the liquid crystal. A polarizer above the liquid crystal layer transmits or absorbs the reflected light, depending on the modulation. There is no voltage applied to the photodiodes during the 

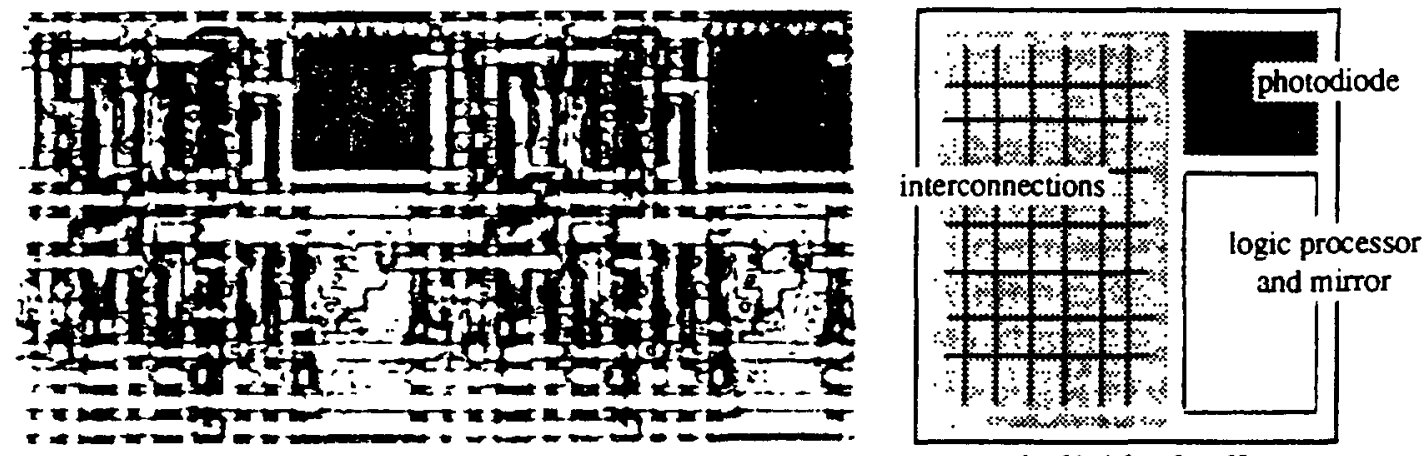

individual cell

Fig. 9. - Photograph and schematic of two cells of a $64 \times 64$ Mathematical Morphology retina.

reading, so the memorized information is not erased. This is particularly interesting, because intrinsic memorized FLC states are not required.

Depositing FLC onto silicon VLSI chips presents several challenges. First, in order to obtain maximum contrast between the two switching states, the liquid crystal must have a thickness corresponding to a $\lambda / 4$ phase delay. One way to achieve this thickness technologically is to use a fixed binding to hold the assembly in place while the glass is being glued onto the chip. Another solution is to use spacers. The achievable optical quality of the device will be limited by the rough surface of the circuit and by the placement of the mirrors. For the chip to be compatible with coherent illumination, the mirror surfaces must be optically flat and co-planar with the chip surface. Another concern is the possibility of false switching of the liquid crystal due to the difference in potential between the circuit and the counter-electrode. Such a problem must be minimized by a proper circuit design. In the present photodiode configuration it has been possible to place the glass directly onto the mirrors, thus using the mirrors as spacers and the photodiode surfaces themselves as the reflectors beneath the liquid crystal layer.

\section{Some domains of applications.}

A large number of applications, based on the use of FLC light valves [37], have been proposed, like a novelty filter [38], the Lyot filter [39], second harmonic generation, and phase conjugation [40]. Another interesting investigation has concerned the implementation of computer generated 2-D phase only hologram recorders [41]. Nevertheless, an exhaustive review is quite impossible. We have decided to focus here on two applications. The first concerns the domain of information processing and more precisely the implementation of realtime Joint Transform Correlator (JTC). A recrudescence of attention has been payed for such an architecture, in the last two years, due principally to device improvements (i.e. in contrat, nonlinearity, ans speed), resulting from the supply of fast FLC light valves and EASLMs [42]. The use of fast switching elements allows a time multiplexing of the references with respect to the video rate. Taking into account a reasonable device development, time multiplexing of 50100 different references for each video scene can be expected, when using, for instance, a silicon backplane EASLM for inputing the references [43]. The second improvement is due to the use of a FLC bistable OASLM (BOASLM) in the Fourier plane. Although the dynamic range of the nonlinear response is limited, experimental results have shown that this feature can be adjusted $[44,45]$. Increasing the severity of the nonlinearity is well known to improve the discrimination capability. Such devices have been successfully used to enhance the correlation peak and therefore to improve the detection performance of standard correlators. 
The second kind of promising applications of FLC light valves concerns the domain of complex systems and neural computing. A review of the main capabilities of FLCs for neural implementation has been presented by Johnson in [46]. Requirements for optical neurocomputing are similar to those of digital optical computing, except that might frame rate are not essential. FLC light valves can be used in neurocomputing as input-output display, storage medium for the synaptic weight as well as nonlinear elements for decision making. The critical step of neural net optical implementations is the learning process and more precisely the weight encoding and updating. To encode weights, grey levels are needed. Although the FLC devices are binary devices, grey levels can be achieved by temporal, and/or spatial multiplexing. A supervised learning algorithm has been implemented in storing the connection weights as pixels in a EASLM [46]. The trade-off is, in this case, between encoding capacity and control.

All optical alternatives exist. Smectic A materials are also very promising in this domain. Because they provide a linear modulation of light in response to an applied field and permit a high switching speed independent of grey levels, smectic A OASLM potentially have all the operating characteristics necessary to build large size fully interconnected neural network architectures with grey level capability [47]. The major problem in this case is related to the unipolar encoding of the weights (because of using a polarization encoding). This has given rise to ingenious solutions using basically two polarization encoding channels $[48,49]$.

Another solution using smectic $C^{*}$ and aiming at taking advantage of the multi-domain switching of BOASLMs to implement the weight encoding and updating has been proposed [50]. In this manner, the value of the grey level may be stored, increased or decreased. The spatial accumulation capability of the device, together with possible thresholding of the image bearing light beam, allows the memorization, accumulation, and erasure of pictures. The device performs basically a logic OR between successive picture exposures. Data can therefore be successively recorded with varying gains. The value of the gain depends on the apparent sensitivity of the device and can be influenced by varying the electrical driving parameters of the BOASLM. In order to store independent weights with a sufficient amplitude range, a minimal FLC area of $100 \times 100 \mu \mathrm{m}$ is necessary. This would allow storage of about 10.000 weights $/ \mathrm{cm}^{2}$ using a conventional BOASLM. Taking into account this capability, a learning process which can be assimilated to the hetero-associative memory principle has been proposed [51], making this approach close to that using photorefractive materials. The difference to the latter is related to the fact that information is spatially stored in a matrix of weights (the BOASLM) instead of being stored in the volume. The BOASLM acts like a photographic plate subjected to multiple exposures and used as a 2-D hetero-associative long term reconfigurable memory.

To be complete, we may mention that FLC light valves can be also used to implement decision steps in neural architectures. A good example can be given by the implementation of the Winner Takes All (WTA) algorithm. Nevertheless, a proper WTA function is well known to be difficult in implementing conventional nonlinear-optical devices (like FLC BOASLM) due to the need of a nonlocal nonlinearity (the output of each unit in an array depends on a nonlinear fashion on the inputs of all the units in the array). The emerging very-large-scale integrated circuit/liquid crystals (VLSI/LC) device technology enables the design of devices with optical inputs and outputs that can be coupled with almost any desired electronic functionality. A noteworthy illustration of such an implementation has been proposed by Slage and Wagner in [52], coupling FLC material with photoaddressable silicon VLSI array. Figure 10 shows a schematic cross-section of two units in a one-dimensional VLSI/FLC WTA array. 


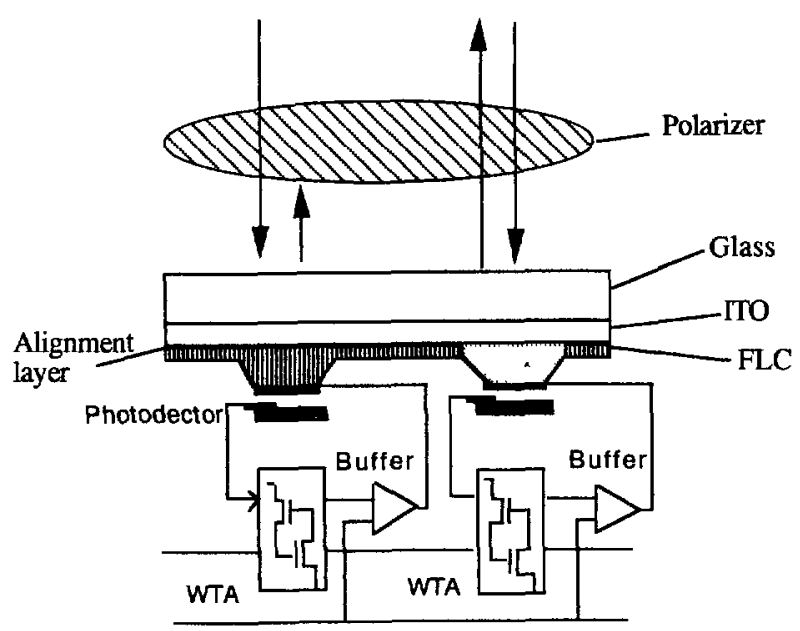

Fig. 10. - Cross-sectional diagram of two units of a VLSI/FLC Winner Take All device (Ref. [49]).

\section{Material and device trends.}

Liquid crystal light valves still remain the crucial element for many optical information processing, communication, and display systems. Progress in the development of light valves, in particular for optical processing applications. has accelerated in the last few years. We also observe the penetration of such SLM technologies into new applications, namely, those of phase conjugation, adaptive optics, and neural network systems. The implementation of real time correlators using fast light valves and SLMs is now a reality. This is due to the arrival of new devices on the market, with better performances and reliability. Therefore, the implementations have overcome the level of the bench prototype and concern now the domain of industrial applications. Advances in the field of optical light valve design have been decisive and contribute directly to the elaboration of a new kind of optical hardware.

We believe that the real challenge lies in our ability to design complex versatile devices such as smart light valves and. more generally, devices coupling electronic and optical functions on a large interconnectable array. It is expected that smart light valves will be the key components of future data processing systems because they can blend the functional flexibility of electronics and the communications and interconnections capabilities of optics. Therefore, we can foresee applications of silicon VLSI/smectic A or $\mathrm{C}^{*}$ optoelectronic devices in areas where massive parallelism and a low power operation at moderate speeds may be suitable, such as special purpose image processing systems, realization of early vision models and optoelectronic neural network. Silicon VLSI/FLC light valves have the great advantage of simplicity of design and fabrication, simplicity of integration with standard electronics, low power requirements, relatively inexpensive fabrication, high frame rates, and the possibility of fabricating large two-dimensional arrays. In this prospect, new generations of light valves based on this principle will bring considerable changes in the field of image processing in the next few years, if we are able to design devices with a satisfying optical quality.

Furthermore, SLMS have suffered both a moderate speed and contrast reduction due to alignment defaults (particularly true with FLC materials). Developments of new materials such as fast smectic A and antiferroelectric liquid crystals (AFLC) [53], with better optical quality than FLCs (i.e. high contrast, less zig-zag defects, etc.) will considerably improve the performances of smectic $A$ and $C^{*}$ LCLVs. Particularly, the use of AFLC will reduce 
considerably the technological constraints. Tri-stable switching shows a double hysteresis loop, satisfying the multiplex driving specification. Moreover, since in the AF state the polarization charges cancel between layers, the « ghost » phenomenon can be avoided. For this reason, the tri-stable switching will argue in favour of strong developments of smart light valves involving low power and high contrast liquid crystal material in the near future. Regarding the problem of the photoaddressing, the development of new photoconductive materials such as a-Ge:H, a-Ge $\mathrm{Si}_{1-1} . \mathrm{H}$ alloys [54], a-Si $\mathrm{i}_{1,} \mathrm{~N}_{1}: \mathrm{H}$ [55], a-Si $\mathrm{i}_{1,} \mathrm{C}_{1}: \mathrm{H}$, their devices and the related technology matched with smart valves will certainly spread application areas of the light valves.

\section{References}

[1] Casasent D., Coherent light valves, Applied Optics and Optical Engineering, R. Kingslake Ed. 6 (New York. Academic, 1979) 143-200.

[2] FISHER A. D.. SLMs : functional capabilities, applications and devices, Int. J. Optoelectron. 5 (1990) 125-167.

[3] Efron U., The silicon liquid crystal light valve, JAP 57 (1985) 1356-1368.

[4] Special Issue of Applied Optics on SLMs for optical information processing 28 (15 November 1989) $4715-4954$.

[5] Special Issue of Applied Optics on SLMs for optical information processing 31 (10 July 1992) 38574072.

[6] BleHa W. P. et al., Application of the liquid crystal light valve to real-time optical data processing, Opt. Eng. 17 (1978) 371-384.

[7] Aubourg P., Huignard J. P., Hareng M. and Mullen R. A., Liquid crystal light valve using bulk monocrystalline BSO as photoconductive material, Appl. Opt. 21 (1982) 3706-3712.

[8] Selected papers on liquid crystal for optics, SPIE Milestone series MS 46 (Bellingham WA, 1992) 1-691.

[9] Patel J. S. and Goodby J. W., Properties and applications of FLC, Opt. Eng. 26 (1987) 373-384.

[10] Wu S. T., EFron U. and Hess L. D., Optical rotatory power of $90^{\circ}$ twisted nematic liquid crystals, Appl. Phys. Lett. 44 (1984) 842-845.

[11] Lohman A. W., Polarization and optical logic, Appl. Opt. 25 (1986) 1594-1597.

[12] DE GenNes P. G., The physics of liquid crystals (Oxford University Press, 1974).

[13] Meyer R. B., Liébert L., Strzelecki L. and Keller P.. Ferroelectric liquid crystal, $J$. Phys. France 36 (1975) 69-71.

[14] Clark N. A. and Lagerwall S. T., Appl. Phys. Lett. 36 (1 June 1980).

[15] DuPONT L. et al., Linear electroclinic coupling in ferroelectric liquid crystals, J. Phys. II France 1 (1991) $831-844$.

[16] Killinger M., DE Bougrenet J. L. and Cambon P., Controlling the grey level capacity of a bistable FLC SLM, Proc. Ferroelectrics 122 (1991) 89-99.

[17] Hartman W. J. A. M., FLC displays for television application, Proc. Ferroelectrics 122 (1991) 355-380.

[18] Killinger M., de Bougrenet J. L., Chittick R. C. and Crossland B., Bistability and nonlinearity in FLC OASLMs : application to neurocomputing, Appl. Opt. 31 (1992) 39303936.

[19] Clark N. A., Rieker T. P. and Maclennan J. E., Ferroelectrics 85 (1988) 79-97.

[20] De Bougrenet de la Tocnaye J. L. and Brocklehurst J. R., Parallel access read/write memory using an optically addressed ferroelectric SLM, Appl. Opt. 30 (1991) 179-180.

[21] Cambon P., de Bougrenet de la Tocnaye J. L. et Killinger M., Modulateurs spatiaux bistables à cristal liquide et à adressage optique pour le calcul optique, J. Phys. III France 1 (1991) 4567. 
[22] Rose A.. Concept in Photoconductivity and Allied Problems (Interscience. New York Academic Press, 1960).

[23] Anderson D. A. and Spear W. E., Philos. Mag. 36 (1977) 695.

[24] Madan A. and Shaw M. P., The physics and Application of Amorphous Silicon (Academic Press Inc, 1988).

[25] Johnson K. M., Flat panel displays or burst, Physics World (September 1992) 37-42.

[26] Tieje T. and Rose A. W., Solid State Commun. 37 (1981) 49.

[27] Crossland W. A. et al., Silicon active backplane SLMs using FLCs, Tech. Digest series, OSA conference on SLM and applications 14 (September 1990) 94-97.

[28] Moddel G., Johnson K. M., Li W. and RICE R. A., High-speed binary optically addresed SLM, Appl. Phys. Lett. 55 (1989) 537-539.

[29] Hebbron M. C. and Makh S. S., Development of Gallium Arsenite based SLM, Proc. Soc. PhotoOpti. Instrum. Eng. 825 (1987) 19-23.

[30] Barbier P.. Walker C. M. and MOddel G.. Suitability of a-Si:H photosensors for optically addressed SLMs, Tech. Digest series, OSA conference on SLM and applications 14 (September 1990) 47-50.

[31] Cambon P., Killinger M. and De Bougrenet de la Tocnaye J. L., Optically addressed SLM with nipin a-Si:H layers and bistrable FLC, Proc. SPIE 1562, Devices for Optical Processing (July 1991) 116-125.

[32] JARED D. and JoHNSON K. M., Optically addressed thresholding very large scale integration liquid crystal SLM, Opt. Lett. 16 (1991) 967-969.

[33] Drabik T. and Handschy M. A., Silicon VLSI/FLC technology for micropower opto-electronic computing devices, Appl. Opt. 29 (1990) 5220-5223.

[34] Devos F., Opérateurs analogiques pour l'intégration de processeurs cellulaires, Colloque interdisciplinaire du CNRS, Technologies matérielles futures de l'ordinateur (Paris 16-17 mars, 1992).

[35] Seyd-Darwish I., Chavel P., Taboury J., Devos F., Raynaud R. and Maurin T., Optoelectronic automata for lattice-gas, Proc. SPIE Optics in Complex systems 1319 (1990) 173 175.

[36] Moignard R., Daniel E., Cambon P. and de Bougrenet de la Tocnaye J. L., Design of a silicon VLSI/FLC smart light valve for parallel optical information processing. Proc. of 8th Workshop SFO on Optics in Computing (Paris 8-9 September, 1992) 3-6.

[37] Johnson K. M., Handschy M. A. and Pagano-STauffer L. A., Optical computing and image processing with ferroelectric liquid crystal, Opt. Eng. 26 (1987) 385-391.

[38] Mao C. C., Johnson K. M., Turner R., JARed D. and Doroski, Applications for binary and analog a-Si:H/FLC OASLMs, Appl. Opt. 31 (1992) 3908-3916.

[39] Masterson H. J., Sharp G. D. and Johnson K. M., Ferroelectric liquid-crystal tunable filter, Opt. Lett. 14 (1989) 1249-1251.

[40] Mao C. C., Johnson K. M. and Moddel G., Optical phase conjugation using optically addressed chiral smectic liquid crystal SLMs, Ferroelectrics 114 (1991) 45-53.

[41] Mok F., Diep J., LiU H. K. and Pasltis D., Real-Time Computer generated hologram by means of LCLV television SLM, Opt. Lett. 11 (1986) 748-750.

[42] JaRed D., Johnson K. M. and MOdDEL G., Joint Transform Correlation using an amorphous silicon FLC SLM, Opt. Commun. 76 (1990) 97-102.

[43] Guibert L., Keryer G., Pellat-Finet P. and de Bougrenet J. L., Compact JTC for roadsign recognition, Proc. SFO on Optics in Computing (Paris, September 8-9, 1992).

[44] Kohler A., Fracasso B., Ambs P. and de Bougrenet de la Tocnaye J. L., JTC using nonlinear FLC SLM, Proc. SPIE 1564 (1991) 236-243.

[45] Hudson T. D. and Gregory D. A.. Nonlinear response of liquid crystal SLMs, Proc. SPIE 1347 (1990) 338-343.

[46] Johnson K. M. and Moddel G., Motivations for using FLC SLMs in neurocomputing, Appl. Opt. 28 (1989) 4888-4899.

[47] Abdulhalim I., Moddel G. and Johnson K. M., High speed analog SLM using a-Si:H and an electroclınic liquid crystal, Appl. Phys. Lett. 55 (1989) 1603-1605.

[48] Robinson M. G., Johnson K. M., Jared D., Doroskı D. and Moddel G., Custom designed 
electrooptic components for optically implemented multilayer neural networks, SPIE Proc. Optical Computing (Salt Lake City, 1990) 84-87.

[49] Gomes C. M., Yamazaki T. and Kobayashi S., Bipolar optical neural networks using FLS devices, Neural Networks 5 (1992) 169-177.

[50] Yao K., Duvillier J., Killinger M. and de Bougrenet de la Tocnaye J. L., Implementation of an optically reconfigurable synaptic weight map using FLC spatial light modulators, Proc. of 8th Workshop SFO on Optics in Computing (Paris, 8-9 September, 1992).

[51] Yao K., Duvillier J., Killinger M. and DE Bougrenet De la Tocnaye J. L., All-optical implementation of a Kohonen-like Map Neural Net with locally distributed continuous Neurons, submitted to special issue of Applied Optics on Neural Networks. 1993.

[52] SLagle T. M. and Wagner K., Winner-take-all spatial light modulator, Opt. Lett. 17 (1992) $1164-$ 1166.

[53] Yuchiro Yamada et al., Ferroelectric liquid crystal display using tristable switching. Jpn J. Appl. Phys. 29 (1990) 1757-1764.

[54] Searle T. M., Jackson W. A. and Austin I. G., J. Non-Cryst. Solids 97 and 98 (1987) 991-998.

[55] MacKenzie K. D., Eggert J. R., Leopold D. J., Lin Y. M. and Paul W., Phys. Rev. B 31 (1985) 2198. 
Classification

Physics Abstracts

$42.30-42.65-42.70 \mathrm{~F}-42.80 \mathrm{~K}$

\title{
Optimization of an ultrafast OASLM using photoexcitations in organic thin films : the incoherent-to-coherent conversion efficiency of spectral concentration
}

\author{
Jean-Michel Nunzi, Fabrice Charra and Nicola Pfeffer \\ Commissariat à l'Energie Atomique, Direction des Technologies Avancées, Léti, Département \\ d'Electronique et d'Instrumentation Nucléaire, Service de Physique Electronique, Saclay, 91191 \\ Gif-sur-Yvette, France
}

(Received 10 December 1992, accepted 4 May 1993)

\begin{abstract}
Résumé. - Les modulateurs spatiaux de lumière à adressage optique sont caractérisés en termes de densité d'éléments de résolution (pixel), de sensibilité à l'exposition et de temps de commutation. Les matériaux optiques non linéaires du troisième ordre sont attrayants afin d'améliorer les performances en termes de densité d'éléments de résolution et de vitesse de commutation. Ils sont comparés à l'aide de figures de mérite appropriées. Le photochromisme picoseconde de certains oligomères conjugués en fait les matériaux prototypes d'un convertisseur incohérent à cohérent ultra-rapide en film mince. La concentration spectrale est la caractéristique clef permettant d'atteindre de grandes sensibilités à l'exposition au niveau moléculaire. Des règles d'ingénierie moléculaire de la concentration spectrale sont proposées. Les oligomères conjugués de polycycles linéaires ont la structure qui convient à l'obtention d'une sensibilité d'exposition de $1 \mathrm{pJ} /$ pixel dans le domaine des fréquences allant $\mathrm{du} \mathrm{MHz}$ au $\mathrm{GHz}$.
\end{abstract}

\begin{abstract}
Optically addressed spatial light modulators are characterized in terms of pixel density, exposure sensitivity and switching time. Third order nonlinear optical materials are attractive in order to improve on pixel density and switching speed. They are compared using appropriate figures of merit. The picosecond photochromism of some conjugated oligomers makes them prototype materials for thin film incoherent to coherent optical converters. Spectral concentration is identified as the key feature which permits spectrally large exposure sensitivities in single molecules. Molecular engineering rules of the spectral concentration are proposed. Conjugated oligomers of linear polycycles have the required structure $1 n$ order to achieve $1 \mathrm{pJ} / \mathrm{pixel}$ exposure sensitivity in the $\mathrm{MHz}$ to $\mathrm{GHz}$ frequency range.
\end{abstract}

\section{Introduction.}

In the field of image and beam processing. there is great need for devices in which a lightintensity profile can be recorded and transformed into a transmission or a phase retardation profile. This is the role of optically addressed spatial light modulators (OASLM) [1]. A special 
class of such devices is the incoherent to coherent optical converter (ICOC) which permits the achievement of input functions in optical image processors based on holographic and Fourier optics. The most trivial representative of this class is the silver-halide photographic recording plate [2]. Usually, a real image is recorded under incoherent illumination on a photographic film, the developed slide then modulates a coherent beam for optical processing of the image. The drawback of silver-halide photography is the delay, close to one minute, between exposure and processing. Different techniques permit the realization of real time incoherent to coherent optical converters. In this paper, we first discuss some material requirements aimed at optimizing an ICOC with the possibilities offered by third-order nonlinear optical $\left(\chi^{(3)}\right)$ materials. We then report on a new feature of the photochromism of organic molecules, spectral concentration, which is exhibited by a class of conjugated oligomers : $\alpha$-oligothiophenes. Spectral concentration appears as the key feature for improved incoherent to coherent conversion efficiency. Physico-chemical analysis of the process is used to define molecular engineering rules which are at the origin of spectral concentration and which permit its practical use. We conclude with prospects towards using organic thin films for ultrafast incoherent to coherent optical conversion purposes.

\section{Materials requirements.}

As in photographic techniques, two functions characterize OASLMs: the exposure or recording and the readout. In usual (commercial) real time OASLMs, recording is performed using a photoconductor in order to optically switch the voltage applied across an electro-optic material which then modulates the readout beam [1]. This is the case in photoconductor - liquid-crystal light valves $[3,4]$. In photorefractive and in self electro-optic devices [5, 6], both functions are performed in the same material. Basically, OASLMs may be characterized by their spatial resolution ( $\mathrm{Ab}$ in cycle/mm), exposure sensitivity per pixel ( $W$ in $\mathrm{pJ} / \mathrm{px}$ ) where the pixel is defined as the smallest resolution element, and switching time ( $\tau$ in $s$ ). Typical properties of OASLMs are displayed in table I.

In order to exploit the parallel addressing capabilities of light, an optimized OASLM should have a spatial resolution limited by diffraction $\left(\mathrm{Ab} \approx \lambda^{-1} \approx 10^{3} \mathrm{cycle} / \mathrm{mm}\right)$. This determines the maximum thickness of the device. Consider the waist of a Gaussian beam at the focal point

Table I. - Typical performances of OASLMs.

\begin{tabular}{|l|c|c|c|c|}
\hline OASLM & Ab (cycle/mm) & $W(\mathrm{pJ} / \mathrm{px})$ & $\tau(\mathrm{s})$ & Ref. \\
\hline Nematic liquid crystal & 60 & 5 & 0.01 & 3 \\
\hline Ferro. liquid crystal & 100 & 0.2 & $10^{-4}$ & 4 \\
\hline Photorefractive crystal & 300 & 100 & 0.1 & 5 \\
\hline Self Electro-Optic & 12 & 180 & $10^{-6}$ & 6 \\
\hline Quantum well etalons & 100 & 100 & $10^{-8}$ & 7 \\
\hline Silver halide films & $10^{3}$ & $10^{-2}$ & 100 & 2 \\
\hline Prospective & $10^{3}$ & 1 & $10^{-6}-10^{-10}$ & \\
\hline
\end{tabular}


of a lens, its radius $w$ is connected to the Rayleigh range $z_{0}$ by $z_{0}=\pi w^{2} / \lambda, \lambda$ being the wavelength. Resolution is thus maximum (Tab. I) when device thickness is $t \leqslant \lambda$.

The speed of charge-transfer OASLMs is limited by carrier drift and capacitance [3-6]. In order to achieve high speed performances in the $10^{6}$ to $10^{10} \mathrm{~Hz}$ range (Tab. I), a recognized route is the use of materials with large third-order nonlinear optical properties such as quantum confined semiconductor heterostructures [7] or organic semiconducting polymers [8]. Those materials are characterized by a susceptibility $\chi^{(3)}$ describing the complex index change $\delta n=2 n_{2} I$ induced on a read beam by a write (pump) beam of intensity $I$ :

$$
\delta n=3 \chi^{(3)} I / 2 \varepsilon_{0} c n^{2}
$$

The complex phase retardation variation induced inside the material is

$$
\delta \varphi=\delta \varphi^{\prime}+i \delta \varphi^{\prime \prime}=2 \pi\left(\delta n^{\prime}+i \delta n^{\prime \prime}\right) t / \lambda .
$$

In order to achieve a contrast ratio of 10 for the device transmission $T=\exp \left(-2 \delta \varphi^{\prime \prime}\right)$, the imaginary part of the phase retardation must reach $\delta \varphi^{\prime \prime} \approx 1.2$. The same $\delta \varphi^{\prime}$ value is large enough to reach the limiting $34 \%$ diffraction efficiency $\eta \approx|\delta \varphi / 2|^{2}$ of thin phase holographic gratings [9]. As $t \approx \lambda$, the required index change necessary for efficient modulation is $\delta n \approx 0.2$.

For $\chi^{(3)}$ materials to be efficient OASLMs, the write process also needs to be optimized. In view of table $\mathrm{I}$, a realistic switching energy is $W \approx 1 \mathrm{pJ} / \mathrm{px}$. With pixels of $1 \mu \mathrm{m}^{2}$, this corresponds to an average energy consumption $I \tau \approx 100 \mu \mathrm{J} / \mathrm{cm}^{2}$. Using equation (1), this sizes the magnitude of materials optical nonlinearity to work as active elements in thin film OASLMs :

$$
\chi^{(3) / \tau}=2 \varepsilon_{0} n^{2} c \delta n / 3 I \tau \geqslant 10^{-3} \mathrm{~m}^{2} \mathrm{~V}^{-2} \mathrm{~s}^{-1}
$$

The nonlinearity is also alternatively expressed in terms of nonlinear index $n_{2}$ [10]. Its usefull range is $n_{2} / \tau \geqslant 0.1 \mathrm{~m}^{2} / \mathrm{J}$. For a $1 \mathrm{~ns}$ switching time, we need $\chi^{(3)} \geqslant 10^{-4}$ esu $\left(1 \mathrm{~m}^{2} V^{-2}=\right.$ $7.2 \times 10^{7} \mathrm{esu}$ ). Such a magnitude is achieved only when optical-field frequencies are close to resonance with real transitions.

In order to evaluate the optical modulation potentialities of nonlinear materials, we must estimate the magnitude of resonant nonlinearities in terms of elementary material parameters. We consider an assembly of non-interacting molecules described by a quantum three-level system. Molecules in the ground state $g$ are excited in resonance using a recording beam at frequency $\omega_{\mathrm{e}}$. The excited state e has a lifetime $\tau$. The induced absorption from the excited state $e$ to the final state $f$ is tested in resonance using a readout beam at frequency $\omega_{\mathrm{i}}$. For a homogeneous isotropic assembly of one-dimensional (rod-shaped) molecules with density $N$, the resonant part of the relevant third-order nonlinear optical susceptibility can be written :

$$
\chi^{(3)}=\operatorname{Im}\left(\chi_{\left(\omega_{\mathrm{e}}\right)}^{(1)}\right) \tau\left(\frac{\left|\mu_{\mathrm{ef}}\right|^{2}}{\omega_{\mathrm{fe}}-\omega_{\mathrm{t}}-i \gamma_{\mathrm{fe}}}-\frac{2\left|\mu_{\mathrm{ge}}\right|^{2}}{\omega_{\mathrm{eg}}-\omega_{\mathrm{t}}-i \gamma_{\mathrm{eg}}}\right) / 10 \hbar^{2}
$$

where $\mu_{1 \mathrm{j}}$ are transition dipole matrix elements and $\gamma_{1 \mathrm{j}}$ transverse relaxation rates between states $i$ and $j$. The linear susceptibility of the material is $\chi^{(1)}\left(\omega_{\mathrm{e}}\right)=N\left|\mu_{\mathrm{ge}}\right|^{2} / 3 \hbar \varepsilon_{0}\left(\omega_{\mathrm{eg}}-\omega_{\mathrm{e}}-i \gamma_{\mathrm{eg}}\right)$. It is related to the absorption of the sample at wavelength $\lambda$ by $\alpha=2 \pi \operatorname{Im}\left(\chi^{(1)}\right) / n \lambda$. The refractive index of organic materials is $n \approx 1.5$ ( $n \approx 3.5$ in GaAs). An intrinsic element of comparison between nonlinear optical materials is thus :

$$
\chi^{(3) / \alpha \tau \approx 3 \varepsilon_{0} n^{2} \lambda^{2} \Delta \sigma / 40 \pi^{2} \hbar}
$$


Its represents the net optical switching efficiency per absorbed photon. It is also the switching efficiency per excited molecule and it is proportional to the average molecular absorption cross-section variation between ground and excited states $\Delta \sigma(\sigma=\alpha / N)$. Using equation (3)

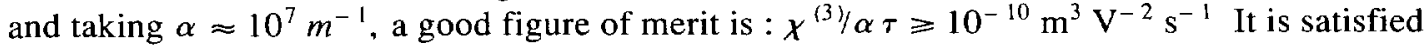
when $\Delta \sigma \geqslant 2 \times 10^{-15} \mathrm{~cm}^{2}$. Table II gives a comparative evaluation of materials tested at resonance. Their switching efficiencies span over 4 orders of magnitude.

Table II. - Switching efficiencies of resonant nonlinear materials obtained from saturable absorption (sa) and photochromism (pc) experiments.

\begin{tabular}{|c|c|c|c|}
\hline Materials & $x^{(3 / / \alpha \tau}$ & $\left.V^{-2} s^{-1}\right)$ & Ref. \\
\hline Cs vapor & 1 & (sa) & 11 \\
\hline $\mathrm{C}_{60}$ & 0.03 & $(\mathrm{pc})$ & 12 \\
\hline Au colloid & 0.4 & & 13 \\
\hline $\mathrm{CdS}_{\mathrm{x}} \mathrm{Se}_{1-\mathrm{x}}$ glass & 0.5 & (sa) & 14 \\
\hline PTS crystal & 80 & (sa) & 15 \\
\hline 9740 Kodak dye & 2 & (sa) & 16 \\
\hline AsGa bulk & 30 & (sa) & 17 \\
\hline AsGa MQW & 300 & (sa) & 18 \\
\hline Thermal effect & 0.1 & & 10 \\
\hline cis-trans isomery & 0.01 & (pc) & 19 \\
\hline Bacteriorhodopsin & 0.05 & (pc) & 20 \\
\hline$\alpha 6$-thiophene & 1 & (pc) & 21 \\
\hline
\end{tabular}

The fully resonant nonlinearities which can be described by simplified three-level schemes are named photochromism in the literature [22]. Few parameters are necessary in order to characterize photochromism in terms of switching efficiency. Large nonlinearities within acceptable switching times and thicknesses are obtained in resonance with fully spin and parity allowed transitions. The efficiency to populate an excited state e depends on the quantum efficiency $\phi$ of conversion of the absorbed photons. Transitions are characterized by their oscillator strengths $f=2 m_{\mathrm{e}} \mu_{\mathrm{lj}}^{2} \omega_{\mathrm{I} J} / e^{2} \hbar$. They are close to unity in most organic dyes. Crosssections of real (inhomogeneously broadened) absorptions are proportional to the inverse linewidth $\Gamma^{-1}$ (hwhm) of the absorption profile. The magnitude of the switching efficiency (5) can then be written as $\chi^{(3) / \alpha \tau}=A \phi f \omega_{\mathrm{l}} / \Gamma_{\mathrm{et}}$ with $A=n c e^{2} / 20 m_{\mathrm{e}} \hbar \omega_{\mathrm{e}} \omega_{\mathrm{t}}^{2}$. For visible $(2 \mathrm{eV})$ to infrared $(1 \mathrm{eV})$ optical conversion, $A=7.8 \times 10^{-13} \mathrm{~m}^{3} \mathrm{~V}^{-2} \mathrm{~s}^{-1}$ The dimensionless figure of 
merit which characterizes efficient photochromic effects is thus

$$
\mathcal{F}=\phi f \omega_{\mathrm{r}} / \Gamma_{\mathrm{ef}} \geqslant 10^{2}
$$

The largest $\mathfrak{F}$ values are obtained with narrow excited state transitions.

\section{Transient photochromism in conjugated oligomers.}

We have performed time resolved pump-probe experiments in order to record the photoinduced (excited-state) absorption spectra of conjugated molecules [21]. The spectra of real and imaginary parts of the anisotropic phase variations photoinduced in an $\alpha$-tertiophene ( $\alpha-3 T)$ solution are represented in figure 1 . The optical density $(6 \delta \varphi / 2.3)$ induced at $\lambda_{\mathrm{t}}=603 \mathrm{~nm}$ is $\approx 0.3$, it is induced with $0.4 \mathrm{~mJ} / \mathrm{cm}^{2}$ excitation at $355 \mathrm{~nm}$ in the maximum $\left(\lambda_{\max }\right)$ of the $\mathrm{S}_{0} \rightarrow \mathrm{S}_{1}$ absorption band. The same spectrum is obtained with an excitation at $396 \mathrm{~nm}$ on the red side of the absorption band. The transient excited state lifetime is 175 ps and we observe the singlet state $S_{1} \rightarrow S_{2}$ transition. The new characteristic feature observed in the transient photochromism of $\alpha-3 \mathrm{~T}$ is the narrow $40 \mathrm{~nm}(0.12 \mathrm{eV}$ FWHM) width of the absorption peak induced at $603 \mathrm{~nm}$ as compared to the broad $65 \mathrm{~nm}(0.6 \mathrm{eV}$ FWHM) linear absorption band peaking at $355 \mathrm{~nm}$ in solution. This effect of spectral concentration between ground and excited state transitions is the required photochromic feature in order to build an ICOC. Indeed, the broad linear absorption ensures broad band exposure sensitivity in order to record images and the narrow transient absorption band ensures efficient readout with coherent light either in absorption or in phase. In the case of $\alpha-3 \mathrm{~T}$, we get $\omega_{\mathrm{t}} / \Gamma_{\text {et }} \approx 30$ in figure 1 and as $\phi f \approx 1$, the photochromic OASLM figure of merit defined by equation (6) is $\mathcal{F}=30$.

$\alpha-n \mathbf{T}$ thiophene oligomers, $n$ being the number of repeat units, have their extinction coefficient $\varepsilon$ increased by one-order of magnitude between ground and transient excited state. Except for $n=4$, the studied oligomers exhibit spectral concentration of the excited state transition. Their photochromic figure of merit is $\mathcal{F} \approx 20-50$. The readout frequency $\lambda_{\mathrm{t}}$ of an $\alpha-n \mathrm{~T}$ ICOC may be adjusted by chosing the oligomer length $n$. In all cases, the formation time of the singlet excited state is smaller than 10 ps and its recovery time is in the

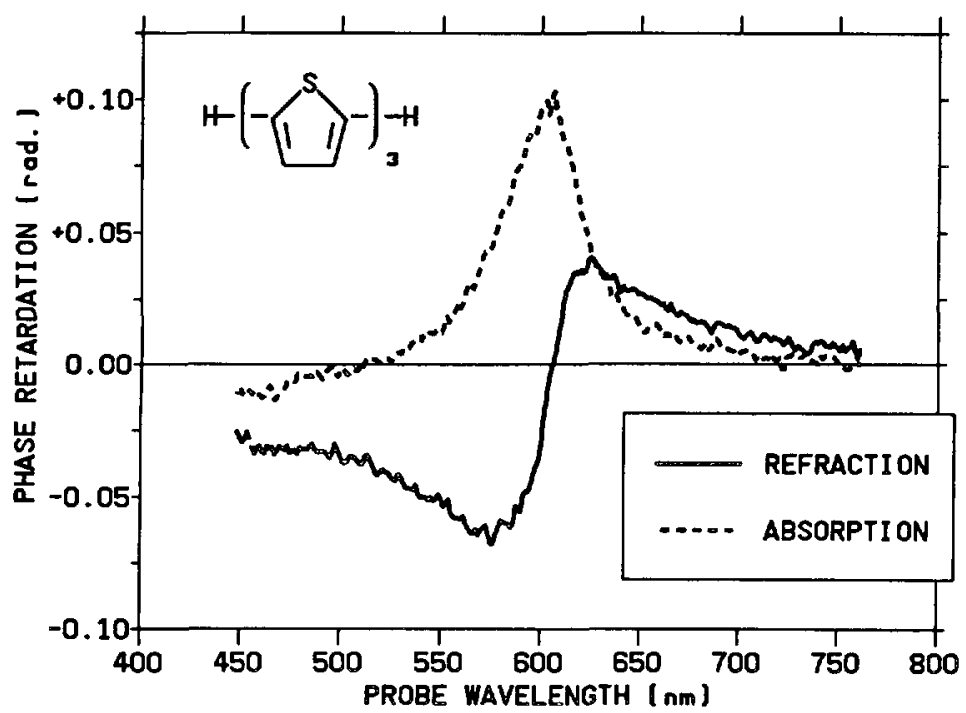

Fig. 1. - Complex phase variations induced in the thiophene oligomer $\alpha-3 T$. 
$100-200 \mathrm{ps}$ range owing to intersystem crossing. Thiophene oligomers are thus potentially efficient candidates as active elements in $10 \mathrm{GHz}$ ICOCs.

The photoinduced absorption of a $\alpha$-6T $3200 \AA$ thin film is represented in figure 2 together with its linear absorption spectrum. The photoinduced absorption characteristics are independent of the pump excitation frequency. The formation time of the transient absorption is close to $50 \mathrm{ps}$ and we measured a recovery time $\tau \approx 5 \mathrm{~ns}$ under room conditions. This excited state is attributed to the lowest $T_{1}$ triplet state of the molecule. Spectral concentration is readily observed in figure 2 where the excited absorption peak has $0.1 \mathrm{eV}$ full half-width and the ground state absorption has $1 \mathrm{eV}$ full half-width. In this film, the estimated OASLM figure of merit is $T \approx 60$, making it a potentially efficient candidate for $200 \mathrm{MHz}$ photochromic ICOCs.

\section{Molecular engineering of spectral concentration.}

Spectral concentration in the excited state absorption results from a series of causes of which three seem essential : (1) damping of vibronic side-bands, (2) conservation of the oscillator strength, and (3) homogeneity of the molecules.

i) Damping of the vibronic side-bands of the ground as compared to the excited state absorption is the most characteristic feature in figure 2. Broad absorption bands are usually a peculiarity of the spectra of organic dyes as opposed to atomic and ionic absorptions. Indeed, many vibrations are closely coupled to the electronic transitions as transitions induce a change in electron densities over the bonds constituting the conjugated chain. This is the case with the $\nu_{\mathrm{v}}=1550 \mathrm{~cm}^{-1} \mathrm{C}=\mathrm{C}$ vibration producing the absorption fingers (vibronic replica) in figure 2. The fingers depend on how many sublevels are reached and how large transition moments to the sublevels are (Franck-Condon factors) [23]. After the excitation has occured $\left(S_{0} \rightarrow S_{1}\right.$ in Fig. 3), there is a change in electron density between carbon atoms. $C=C$ bonds are lengthened and the new equilibrium position becomes $\delta r$ in figure 3 . In a classical picture, carbon atoms start to oscillate around the new position with an amplitude $\delta r$. The equilibrium position is usually reached within $10^{-12} \mathrm{~s}$. Narrowing of the photoinduced absorption in figure 2 comes essentially from the damping of Franck-Condon factors to the $\nu_{\mathrm{v}}\left(1500 \mathrm{~cm}^{-1}\right)$ sublevels in the excited state. As pictured in figure 3, this means that the equilibrium positions of the carbon atoms are not modified in the excited state absorption $S_{1} \rightarrow S_{2}$ (or $\mathrm{T}_{1} \rightarrow \mathrm{T}_{2}$ ). In order for equilibrium positions of carbon atoms to remain unchanged by electronic transitions, there must be only weak localization of the long single $\mathrm{C}-\mathrm{C}(\approx 1.45 \AA)$ and short

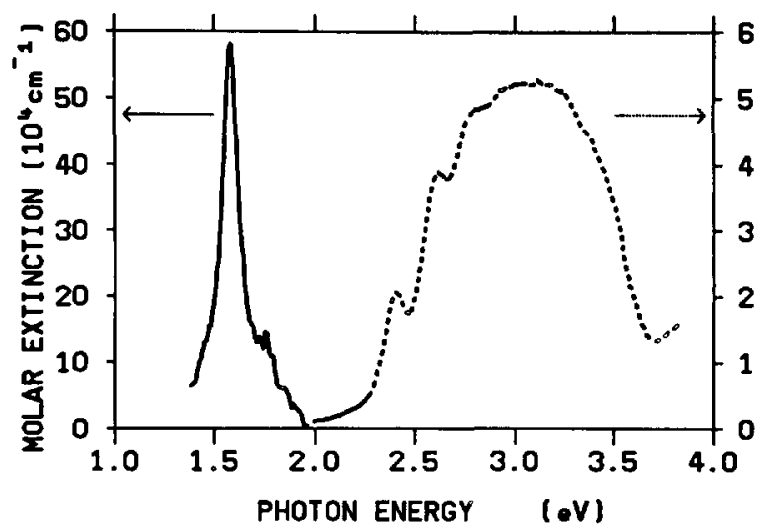

Fig. 2. - Linear (- -) and photoinduced (-) absorption in $\alpha-6 \mathrm{~T}$ thin films. 


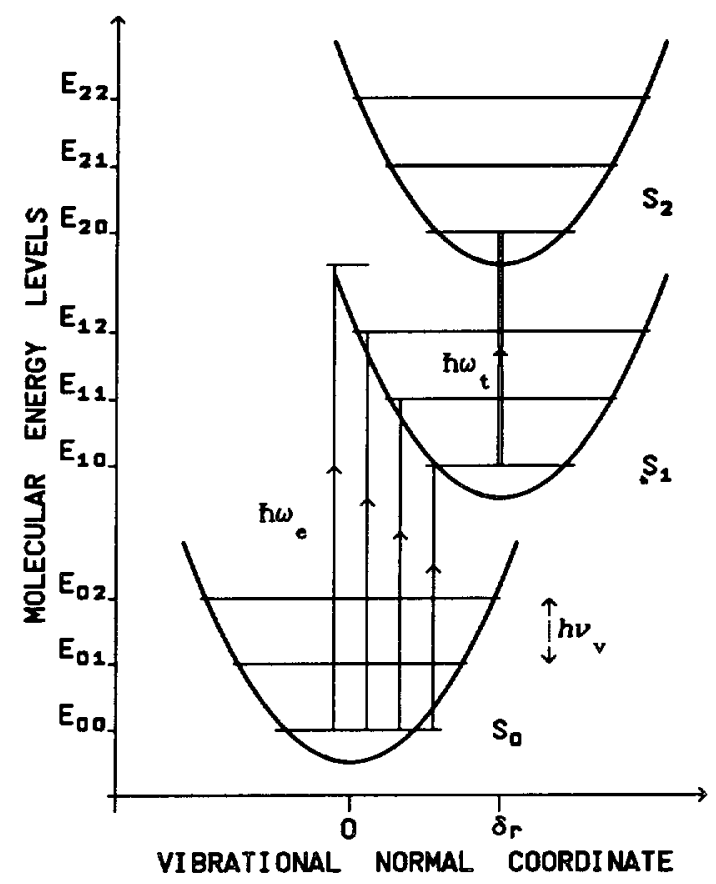

Fig. 3. - Transitions between molecular energy levels. The nuclear potential surfaces are projected along the normal coordinate of vibration. the vibronic side bands after transition to the excited state are damped.

double $\mathrm{C}=\mathrm{C}(\approx 1.35 \AA$ ) bonds along the carbon skeleton; that is that bond lengths do not alternate in the excited state as much as they do in the ground state of thiophene oligomers.

The reduction of bond alternance can be seen as a general feature of the excited state of symmetric conjugated molecules with an even number of carbon atoms in a polyene-like skeleton (Fig. 4). Indeed, their lowest energy transition is a $\pi \rightarrow \pi^{*}$ transition which keeps the symmetry of the molecule. The excited molecule being non-polar, as observed in $\alpha-3 T$ [21], the excess electron pair has to be distributed symmetrically along the conjugated skeleton, thus reducing the length difference between single and double bonds. Such excited-state electron distribution is accounted for in the simplest Hückel molecular orbital theory in which the number of nodes (zeros) of the molecular orbitals increases with the energy, as do free-electron wave-functions in a quantum box [23]. Molecules in which the relative motion of carbon atoms is hindered by a ladder structure (such as condensed rings) [22] have poor performances in this respect [24]. The engineering rule for the damping of vibronic side-bands is thus to use

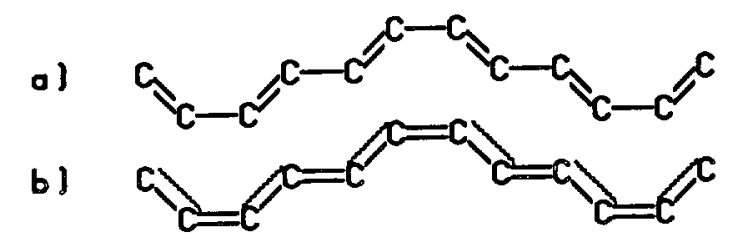

Fig. 4. $-\pi$ electron distribution along the conjugated carbon skeleton of the $\alpha-3 \mathrm{~T}$ oligomer in the ground (a) and lowest excited state (b). Dashed lines in (b) represent a symmetric repartition of the two unpaired electron which are in excess as a result of the $\pi \rightarrow \pi^{*}$ transition. 
molecules with a conjugated polyene-like skeleton having the possibility of bond-length change upon optical excitation.

ii) Conservation of the oscillator strength is typical of the spectral concentration achieved in figure 2 in which the absorption coefficient $\alpha$ is increased by a factor of 10 between ground and excited states. The sum rule states that over the whole UV-IR spectrum, absorption verifies

$$
\int \alpha(\omega) \mathrm{d} \omega=\text { Cte } .
$$

In order for the relevant photoinduced transition to satisfy equation (7), it is necessary to have a large quantum efficiency $\phi$ of transient excited state formation. By nature, singlet $\left(S_{1}\right)$ states verify $\phi=1$. Their lifetimes are in the 0.1-100 ns range. Triplet states $\left(\mathrm{T}_{1}\right)$ may also have large population efficiencies $\phi \approx 1$ from the $S_{1}$ state (intersystem crossing). Large quantum efficiencies of triplet state formation are found in aromatic molecules [25]. Their lifetimes span the $100 \mathrm{~ns}-100 \mathrm{~ms}$ range.

The lowest energy fully spin and parity allowed electronic transition in conjugated dyes has an oscillator strength $f \geqslant 1$. Long symmetric conjugated molecules such as polythiophenes have $C_{2 \mathrm{~h}}$ symmetry. The ground state $\mathrm{S}_{0}$ wave function is usually ${ }^{\prime} \mathrm{A}_{\mathrm{g}}$ (even with respect to inversion). The lowest excited $S_{1}$ state is ${ }^{1} B_{u}$ and the second $S_{2}$ excited state has the same ${ }^{1} \mathrm{~A}_{\mathrm{g}}$ symmetry as the ground state. The most coupled states are usually the closest in energy because the number of nodes of their wave functions differ only by one unit. Calculations performed in polyenes up to the octatetraene [26] show that the oscillator strength of the $S_{1} \rightarrow S_{2}$ transition is a factor of 4 larger than that of the $S_{0} \rightarrow S_{1}$ transition. We measured the characteristics of the lowest two-photon state of poly(3-octylthiophene) and we indeed found that the oscillator strength of the $S_{1} \rightarrow S_{2}$ transition is $f \approx 5$ [27]. An engineering rule to achieve large excited-state oscillator strengths is to use long conjugated molecules with onedimensional symmetry.

iii) Homogeneity of the molecules is a necessity in order to reach an excited state in which all absorptions are at the same frequency. This rules out the use of polymers which have not a well defined effective conjugation length. Polymers have photoinduced spectra which cover the visible spectrum as would do mixtures of oligomers. This is the case of polydiacetylene [28] though on the other hand it is a highly nonlinear material (Tab. II) [15].

Simple polyenes often undergo cis-trans isomerisation upon photoexcitation [22]. This can be overcome using cyclic monomers such as phenyls, thiophenes and pyrroles. They show a large ground-state conformational disorder of the inter-ring angle $\theta$ owing to free rotation around the single bond (Fig. 5) [29]. $\pi \rightarrow \pi^{*}$ transitions roughly correspond to a motion of the double bonds from the cyclic to the quinoid form in figure 5 . This inhibits free inter-ring rotation, thus increasing the homogeneity of the excited state structure. Beside lattice and environment effects, an engineering rule for the homogeneity of the excited state transition is thus to use relatively short, up to $n \approx 10$, and regular linear oligomers made of cyclic monomers.
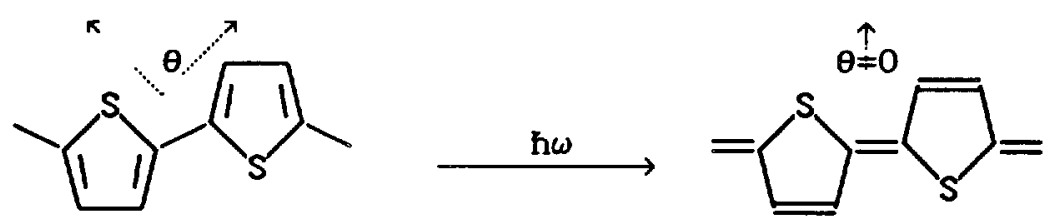

Fig. 5. - Excitation of the non-planar cyclic form to the planar quinoid form in polythiophene. 


\section{Prospects and conclusions.}

More experiments are needed to determine, to control and to remove deleterious effects such as photodegradation and instability of the films. However, during our picosecond pump-probe experiments, materials exhibited good resistance. In any case, an isolated molecule can withstand more than $10^{6}$ excitation-relaxation cycles without degradation [30]. Under $100 \mathrm{~W} / \mathrm{cm}^{2}$ average exposure, the lifetime of a film may thus be larger than $10 \mathrm{~s}$. Moreover, as plastic films can be wound and unwound as magnetic tapes, photodegradation could be removed by cycling as in liquid organic laser dyes.

On the one hand, realization of organic OASLMs using thin films in which optical modulation is achieved on a molecular scale opens the door to devices processing large densities of parallel information (images). On the other hand, organic materials have an ultrafast response time and a relaxation time in the ns to $\mu$ s range, opening the door to optical image processing at $\mathrm{MHz}$ to $\mathrm{GHz}$ frequencies. In this respect, spectral concentration permits achievement of the highest possible optical exposure sensitivities without using amplification nor etalon effects. Knowledge of the molecular engineering rules of spectral concentration will ease the tailoring of molecules with optimized switching time and exposure-readout frequencies. A future target is typically to handle diode laser beams either in amplitude or in phase, to tune the excited state lifetime in order to match the studied transients, while keeping white-light exposure sensitivities within the $1 \mathrm{pJ} / \mathrm{pixel}$ level. For instance, control of the switching time of the OASLM would permit handling of the $800 \mathrm{~nm}$ beam of a diode laser under day-light exposure. The required switching (or integration) time would have to be close to $1 \mu \mathrm{s}$ with $\approx 100 \mathrm{~W} / \mathrm{cm}^{2}$ illumination on the film. It must be mentioned that owing to energy consumption and dissipation problems, $\mathrm{GHz}$ modulation rates would have to be limited to specific ultrafast transient analysis problems. Meanwhile, $\mathrm{MHz}$ rates are within the limits of low switching energy OASLM materials (Tab. I). In this respect, triplet states of organic molecules which have relaxation rates in the $\mathrm{kHz}$ to $\mathrm{MHz}$ range at room temperature are promising candidates. Thiophene oligomers exhibit spectral concentration, they have photochromic performances which make them prototypes of active elements in optimized ultrafast incoherent to coherent optical converters.

\section{Acknowledgements.}

This work was supported by the.Direction des Recherches, Etudes et Techniques. We thank Doctor J. M. C. Jonathan for helpfull discussions. We also thank Doctor D. Fichou for his collaboration on thiophene oligomer studies.

\section{References}

[1] Vanderlugt A., Optical signal processing (Wiley, New York, 1992).

[2] BiedermanN K., Silver halide photographic materials, Holographic recording materials, H. M. Smit Ed. (Springer, Berlin, 1977) p. 21.

[3] Grinberg J., Jacobson A., Bleha W., Miller L., Fraas L., Boswell D. and Myer G.. A new real time non coherent to coherent light image converter : the hybrid field effect liquid-crystal light valve, Opt. Eng. 14 (1975) 217.

[4] Johnson K., Flat panel displays or bust? Phys. World 5 (1992) 37.

[5] Yu J. W., Psaltis D., Marrakchi A., Tanguay A. R. Jr. and Johnson R. V., The photorefractive incoherent to coherent optical converter, Photorefractive materials and their applications II, $\mathbf{P}$. Gunter and J. P. Huignard Eds. (Springer, Berlin, 1989) p. 275. 
[6] Livescu G., Miller D. A. B., Henry J. E., Gossard A. C. and English J. H., Spatial light modulator and optical dynamic memory using a $6 \times 6$ array of self-electro-optic-effect (SEED) devices, Opt. Lett. 13 (1988) 297.

[7] Sfez B. G., Rao E. V. K., Nissim Y. I. and Oudar J. L., Operation of nonlinear GaAs/AlGaAs multiple quantum well microresonators fabricated using alloy-mixing techniques, Appl. Phys. Lett. 60 (1992) 607.

[8] Kajzar F., Messier J., Nunzi J. M. and Raimond P., Third-order materials : processes and characterization, Polymers for Lightwave and Integrated Optics : Technology and Applications, L. A. Hornak Ed. (Marcel Dekker, New York, 1992) p. 595.

[9] Sмiтн H. M., Basic holographic principles, Holographic recording materials, H. M. Smith Ed. (Springer, Berlin, 1977) p. 1.

[10] Stegeman G. I., Nonlinear optical devices : current status of organics, Organic materials for Nonlinear Optics II, R. A. Hann and D. Bloor Eds., Special publication of the Royal Society of Chemistry (London, 1991) p. 311.

[11] Oria M.. Bloch D., Fichet M. and Ducloy M.. Efficient phase conjugation of a cw low-power laser diode in a short cesium (Cs) vapor cell at $852 \mathrm{~nm}$, Opt. Lett. 14 (1989) 1082.

[12] Palit D. K., Sapre A. V., MitTal J. P. and RaO C. N. R., Photophysical properties of the fullerenes, $\mathrm{C}_{60}$ and $\mathrm{C}_{70}$, Chem. Phvs. Lett. 195 (1992) 1.

[13] Hache F., Ricard D., Flytzanis C. and Kreibig U.. The optical Kerr effect in small metal particles and metal colloids : the case of gold, Appl. Phys. A 47 (1988) 347.

[14] Roussignol P., RiCARd D. and Flytzanis C., Nonlinear optical properties of commercialsemiconductor doped glases, Appl. Phys. A 44 (1987) 285.

[15] Greene B. I., Orenstein J., Millard R. R. and Williams L. R., Nonlinear optical response of excitons confined to one dimension, Phys. Rev. Lett. 58 (1987) 2750.

[16] KOECHNER W., Solid-state laser engineering (Springer, Berlin, 1976) p. 465. $\chi^{(3) / \alpha \tau}=$ $\varepsilon_{0} n^{2} c \lambda / 3 \pi \tau I$, in saturable absorbers with saturation intensity $I$, and recovery time $\tau$.

[17] Oudar J. L., Transient nonlinear optical effects in semiconductors, Nonlinear optics materials and devices. C. Flytzanis and J. L. Oudar Eds. (Springer, Berlin. 1986) p. 91.

[18] Chemla D. S., Nonlinear interactions and excitonic effects in semiconductors quantum wells, Nonlinear optics materials and devices, C. Flytzanis and J. L. Oudar Eds. (Springer. Berlin, 1986) p. 65.

[19] Lessard R. A., Couture J. J. A. and Galarneau P., Application of third-order nonlinearities of dyed PVA to real time holography, Nonlinear Optical effects in organic polymers, J. Messier, F. Kajzar, P. Prasad and D. Ulrich, NATO ASI Series E 162 (Kluwer. Dordrecht, 1991) p. 343.

[20] Thoma R., Hampp N., Brauchle C. and Oesterhelt D., Bacteriorhodopsin films as spatial light modulators for nonlinear-optical filtering, Opt. Lett. 16 (1991) 651.

[21] Charra F., Fichou D., Nunzi J. M. and Pfeffer N., Picosecond photoinduced dichroism in solutions of thiophene oligomers, Chem. Phys Lett. 192 (1992) 566. Cross-section $\sigma$ in $\mathrm{cm}^{2}$ and $\varepsilon$ in liter $/\left(\mathrm{cm}\right.$. mole) verify $\sigma=0.385 \times 10^{-20} \varepsilon$.

[22] Dessauer R. and Paris J. P., Photochromism, Advances in Photochemistry, 1 (Interscience, New York, 1963) p. 275.

[23] SALEM L., The molecular orbital theory of conjugated systems (Benjamin, New York, 1966) p. 333.

[24] Beeson K. W., Yardley J. T. and Speiser S., All optical switching by organic nonlinearly absorbing molecules, Nonlinear optical properties of organic materials III, F. Khanarian Ed., SPIE proc 1337 (1990) 364.

[25] Wilkinson F., Triplet quantum yields ans singlet-triplet intersystem crossing, Organic molecular photophysics, J. B. Birks Ed. 2 (Wiley, New York, 1975) p. 95.

[26] Heflin J. R., Wong K. Y., Zamani-KhamiRi O. and Garito A. F., Nonlinear optical properties of linear chains and electron-correlation effects, Phys. Rev. B 38 (1988) 1573.

[27] Pfeffer N.. Raimond P., Charra F. and Nunzi J. M., Determination of the two-photon absorption spectrum of a soluble polythiophene, Chem. Phys. Lett 201 (1993) 357.

[28] Charra F. and Nunzi J. M., Relaxation of one- and two-photon excitations in a polydiacetylene red form : a frequency and phase resolved analysis, Organic molecules for nonlinear optics and 
photonics, J. Messier, F. Kajzar, P. Prasad Eds., NATO ASI Series E 194 (Kluwer, Dordrecht, 1991), p. 359.

[29] Lopez-Navarrete J. T., Tian B. and Zerbi G., Chain flexibility in polyheteroatomic polymers : electronic properties, structure and vibrational spectra of oligomers as models of polypyrrole and polythiophene. Synth. Metals 38 (1990) 299.

[30] RaUe R. and HARNISCH H., Dyestuff lasers and light collectors : two new fields of application for fluorescent heterocyclic componds, Heterocycles 21 (1984) 167. 